
available under aCC-BY-NC-ND 4.0 International license.

\title{
Bi-directional Modeling Between Cross-Scale Neural Activity
}

\author{
Yin-Jui Chang ${ }^{1}$, Yuan-I Chen ${ }^{1}$, Hsin-Chih Yeh ${ }^{1,5}$, Jose M. Carmena ${ }^{2,3}$, Samantha R. Santacruz ${ }^{1,4}$ \\ ${ }^{1}$ Biomedical Engineering, University of Texas at Austin, Austin, TX, USA \\ ${ }^{2}$ Electrical Engineering \& Computer Sciences, University of California, Berkeley, Berkeley, CA, USA \\ ${ }^{3}$ Helen Wills Neuroscience Institute, University of California, Berkeley, CA, USA \\ ${ }^{4}$ Institute for Neuroscience, University of Texas at Austin, Austin, TX, USA \\ ${ }^{5}$ Texas Materials Institute, University of Texas at Austin, Austin, TX, USA
}

\section{Abstract}

Fundamental principles underlying computation in multi-scale brain networks illustrate how multiple brain areas and their coordinated activity give rise to complex cognitive functions. Whereas the population brain activity has been studied in the micro- to meso-scale in building the connections between the dynamical patterns and the behaviors, such studies were often done at a single length scale and lacked an explanatory theory that identifies the neuronal origin across multiple scales. Here we introduce the NeuroBondGraph Network, a dynamical system incorporating both biological-inspired components and deep learning techniques to capture cross-scale dynamics that can infer and map the neural data from multiple scales. We demonstrated our model is not only 3.5 times more accurate than the popular sphere head model but also extracts more synchronized phase and correlated low-dimensional latent dynamics. We also showed that we can extend our methods to robustly predict held-out data across 16 days. Accordingly, the NeuroBondGraph Network opens the door to revealing comprehensive understanding of the brain computation, where network mechanisms of multi-scale communications are critical.

\section{Introduction}

Billions of individual neurons coordinate activities by employing system-level dynamics to drive behaviors such as motor preparation ${ }^{1,2}$, motor adaptation ${ }^{3}$, motor timing ${ }^{4,5}$, decision-making ${ }^{6}$, and working memory ${ }^{7,8}$. Current techniques for capturing neural dynamics by extracting low-dimensional latent processes typically make simplified assumptions in which dynamics are linear ${ }^{9}$ or log-linear ${ }^{10}$, and work to date has largely focused on measurements from a single modality. While within-level, nonlinearly correlated neural dynamics encode rich information giving rise to behavior ${ }^{11}$, cross-level nonlinear communication, which uncovers a deeper understanding of system-level neural mechanisms ${ }^{12,13}$, is seldom explored. Since the brain exhibits computational structure across a variety of scales: from single neurons (micro-scale) to functional areas (meso-scale) to cortical networks (macro-scale), a tool that can uncover multi-scale dynamics is critically important for illuminating the mechanistic understanding of brain computation.

Recent cross-level analyses include source localization and cross-level coupling $(C L C)^{14}$. Source localization is to identify the brain areas or individual neurons generating the recorded electrical potential ${ }^{15}$. However, the requirement of high-density recordings, unrealistic assumptions, and uncertainty on conductivity value ${ }^{16}$ limit the fidelity on experimental data. Although CLC method has shown the evidence of interactions, no information about how the activity communicates across levels was provided. Here we introduce a multi-scale dynamical model, termed the NeuroBondGraph Network $(\text { NBGNet })^{17}$, to capture the interactions between neurons or networks at disparate scales . Our NBGNet is adapted from a Bond Graph (BG), a modeling framework used to investigate how energy is transferred among different system components $^{18}$, and has been adopted for system identification and fault diagnosis ${ }^{19}$. In the NBGNet, a recurrent neural network (RNN) is constructed based on a priori knowledge obtained from a BG-derive dynamic systems, enabling us to 
approximate the nonlinear mapping to characterize the implicit relations of multi-scale brain activity. Compared to source localization, we bypass the issue of unrealistic assumptions which lead to inaccurate predictions. Unlike purely data-driven methods, the NBGNet is interpretable to evaluate both within- and cross-scale causal interactions.

The NBGNet model can be used for any combination of neural activity at different scales (or even the same scale) with the appropriate selection of the BG. In this work, we characterize our method with two types of simultaneously recorded real data. Namely, we use here local field potentials (LFPs) and signals recorded from screw type electrodes implanted in the skull (screw electrocorticography or screw $E C_{0}{ }^{20,21}$ ), acquired from a rhesus macaque performing a center-out joystick task. Screw ECoG, rather than electroencephalography, is chosen due to its improved signal-to-noise ratio and stability. We demonstrate that the presented method performs more accurately in both the forward and inverse problem compared to other techniques. Low error (3.5-fold improvement) and strong similarity (2.2- and 1.6-fold increase) in both time- and phase-domain to ground-truth signals validate the NBGNet as an accurate solution. Furthermore, the NBGNet can capture and accurately reconstruct single-trial low-dimensional neural dynamics. Behavioral variables can also be predicted by NBGNet-inferred activity as accurately as using empirical measurements. Finally, we examine the stability of the performance of the model and reveal that the learned dynamical system maintains the predictive power over several weeks.

\section{Results}

Overview of NBGNet. To introduce the NBGNet (Fig. 1), we start with a generic dynamical system, where the evolution of latent variables and the output is described by the nonlinear functions of latent states and corresponding input. Nonlinearity is derived from the BG based on the translation between LFP and screw ECoG (Supplementary Fig. 1). Multilayer perceptron units are adopted to approximate the nonlinear functions, generating a sparse RNN, NBGNet, which maximizes the likelihood of the observed brain signals with its internal states (Supplementary Fig. 2).

The forward solution, forward-NBGNet, models the single-trial screw ECoG as a nonlinear recursive mapping from the LFP (Fig. 1a-b). The network's units to approximate such a mapping depend on three elements: a trial-specific initial state, input signals, and the parameters defining the connections of the model. To mimic the real-time modeling and abide by causality constraints, the network only runs through the trial backward for estimation. The inverse model, inverseNBGNet, is then developed by inverting forward-NBGNet. The resulting network predicts LFP across time from the temporal sequence of screw ECoG (Fig. 1a). As inverse computation is an ill-posed problem which can lead to non-unique and unstable solution ${ }^{22}$, we expect a relatively poorer performance when compared with the forward model. However, NBGNet still provide accurate and reliable estimations.

We trained the NBGNet on simultaneously recorded LFP data from the left hemisphere and screw ECoG data from both hemispheres. This data was recorded while a monkey performed a center-out joystick task (Fig. 1c-d; Supplementary Table 1-2). We chose 16 LFP channels and 7 screw ECoG channels from distinct areas to obtain a subset of anatomically spatially distributed signals. The monkey began each trial by holding a cursor at the center target. Then, one of eight outer targets was presented on the screen. After $333 \mathrm{~ms}$, the monkey was instructed to move the cursor to the target for rewards. LFP data was recorded using a semichronic multielectrode array (GrayMatter Research, Bozeman, MT) and screw ECoG data was recorded using screw-type electrodes. The length of each trial is variable, but on average is $1.53 \pm 1.22$ seconds (mean \pm s.d.; Fig. 1e).

We next analytically validated NBGNet-inferred neural activities by calculating root mean squared errors (Fig. 1f-g); reproducing features seen in common neuroscientific analyses (cross-correlation; Fig. 2, phase synchrony; Fig. 3); 
reconstructing low-dimensional latent dynamics (Fig. 4); inferring details of behavior (Fig. 5); and predicting out-of-sample conditions (Fig. 6). For all results in this paper, we trained NBGNets without any information about task conditions or behavioral parameters (e.g., real kinematics or eye-tracker data).

Validation of NBGNet predictions using a center-out joystick task. The representative dataset used in Fig. 1 consisted of 150 individual reach trials (average 175 trials/session). Since the beta frequency band $(12.5-30 \mathrm{~Hz})$ is strongly implicated in motor behaviors ${ }^{23,24}$, (Supplementary Fig. 3), we examined performance with root mean squared error (RMSE) for the predicted broadband signals as well as specifically for the beta band activity extracted from the predictions (Fig. 1f). Long short-term memory based recurrent neural network (RNN) was utilized as baseline for quantitative comparison. NBGNetreconstructed screw ECoG had better match with the ground-truth screw ECoG (RMSE $=0.21 \pm 0.09$ for broadband; 0.13 \pm 0.07 for beta band; mean \pm s.d. in the unit of $10^{-4} \mathrm{~V}$ ) than RNN's predictions (RMSE $=0.28 \pm 0.09$ for broadband; $0.16 \pm$ 0.07 for beta band). Additionally, session-averaged RMSE ( 0.45 for NBGNet $<0.47$ for RNN in broadband; 0.15 for NBGNet $<0.18$ for RNN in beta band; Supplementary Fig. 4) indicated that forward-NBGNet successfully estimated the neural oscillations recorded from screw ECoG.

We also tested whether inverse-NBGNet could recover LFP with screw ECoG recordings (Fig. 1g). Similarly, inverseNBGNet (RMSE $=0.29 \pm 0.12$ for broadband; $0.18 \pm 0.10$ for beta band) outperformed RNN (RMSE $=0.35 \pm 0.13$ for broadband; $0.22 \pm 0.10$ for beta band). Evidenced with the fact that session-averaged RMSE from NBGNet (0.56 for broadband; 0.21 for beta band) was less than that from RNN ( 0.57 for broadband; 0.25 for beta band), inverse-NBGNet was able to transform the lower-dimensional screw ECoG into the higher-dimensional LFP.

NBGNet outputs correlate with ground truth signals. Similarity of oscillation dynamics is another evaluation of integrity of predicted signals using cross-correlations computed on a single-trial single-channel basis. From the representative session (Fig. 2a-b), model-predicted signals from all the channels, excluding channel 1 (S1, right hemisphere), were moderately to strongly correlated with the ground-truth signals with average correlation greater than $0.4^{25,26}$. Strong correlation (correlation coefficient $>0.6$ ) was found on $57 \%$ of channels. Interestingly, due to movement-induced activation, channels at anterior brain regions exhibited larger correlation than those at posterior brain regions. In the representative trial, the predicted screw ECoG matched well with the raw screw ECoG for channel 2 (Fig. 2c). The grand average also indicated that forward-NBGNet accurately reconstructed screw ECoG with consistently small error trace (Fig. 2d). We noted that in channel 7, the performance was relatively poor in the first $0.5 \mathrm{~s}$ when the subject was searching for the correct direction of cursor's movement; however, the performance became better in the rest of time when the direction of movement aligned with the target direction.

We also studied the correlation between the inverse-NBGNet-inferred LFPs and the ground-truth LFPs. Channel 14 (SMA) provided the highest correlation as $0.83 \pm 0.10$ (mean \pm s.d.; Fig. 2e-f). Due to unexpectedly larger amplitude, channel 5 (PMd) exhibited no correlation $(0.02 \pm 0.31)$. However, the predicted signals on the rest of the channels, excluding channel 11 (M1), were at least moderately correlated with ground-truth activity. $44 \%$ of channels showed strong correlations. As more ventral brain region were further from the surface where screw ECoG was recorded, the channels in these regions (channel 11, 13, 15, and 16) showed smaller correlations. In contrast, accurate prediction at channel 14 was expected (Fig. 2g-h). Both single-trial- and grand average-based evaluations demonstrated small deviations between the predictions and the ground truths. Taken together, the correlation analysis confirmed the NBGNet's ability to capture the beta-frequency dynamic features. 
Phase agreement in beta band during movement. Phase-domain coherence is an important tool to determine the integrity of the functional connectivity in brain networks. Accordingly, we examined whether the predicted and the recorded signals were phase-synchronized. Phase-locking value (PLV) has been widely used to measure the inter-trial variability of phase difference, where 1 represents no change in phase difference and 0 reflects the opposite case ${ }^{27,28}$. To assess the intra-trial variability, we adapted PLV by averaging the phase difference across the time rather than the trials (Fig. 3a,e). The phase of phase-locking, which indicates the average phase difference, was taken into consideration as well. Instantaneous phase synchrony (IPS) was then used to measure the phase similarity at each time point. Here we utilized a phase synchrony index (PSI) metric which was determined by the fraction of the time when the IPS was greater than a threshold at which the phase difference was less than $45^{\circ}$ (threshold $=0.62$; Supplementary Fig. 5). If the phase difference seldom exceeds $45^{\circ}, \mathrm{PSI}$ is close to 1 ; it is close to zero otherwise. Forward-NBGNet-predicted signals were in sync with the ground truths ( $73 \%$ of average phase difference $<22.5^{\circ}$; average PLV $=0.62$; average PSI $=0.60$; Fig. $3 \mathrm{~b}$ ). A representative channel, channel 2, was demonstrated to generate phase-synchronized predictions (PSI = 0.74; Fig. 3c). Since PLV and PSI provided disparate aspects, we assessed the phase synchrony for each channel and each trial in the form of a scatter plot (Fig. 3d). Segmentation of the scatter plot enabled us to study the further details (Supplementary Fig. 6). 78\% of predictions exhibited moderate or strong phase synchronization. We next evaluated the inverse-NBGNet's ability to infer the synchronous LFPs. The predictions were in sync with the ground-truth LFPs (62\% of average phase difference $<22.5^{\circ}$; average PLV = 0.56; average PSI = 0.55; Fig. 3f). Highly synchronized predictions at a representative channel, channel 14, were observed (PSI = 0.84; Fig. $\mathbf{3 g}$ ). Furthermore, approximately half of the predictions had strong synchronization (Fig. 3h). Our phase analysis comprehensively validated that the model predictions are phase-synchronized with the ground truth.

NBGNet captures latent dynamics. Since low-dimensional latent dynamics have been widely used to illuminate the relationship between neural population activity and behavior ${ }^{29-33}$, we also tested whether NBGNet could capture latent dynamics. The window of interest starting from movement onset and ending $600 \mathrm{~ms}$ after movement onset was selected, and there is no issue of imbalance target directions (Fig. 4a). We computed the neural manifold and the latent dynamics within it using principal component analysis $(P C A)^{34,35}$. The resulting three PCs, termed neural modes, captured the majority of the variance (Supplementary Fig. 7), and were used to define the axes of the neural manifold. Canonical correlation analysis (CCA) ${ }^{36-38}$ was performed to align the latent dynamics (Fig. 4b). Correlation analysis (Pearson's $r$ ) was then utilized to quantify the similarity of these latent dynamics. Since canonical correlations were sorted from largest to smallest, we expected the same trend in the evaluation.

Representative single-trial and grand-average latent trajectories for ground-truth and reconstructed screw ECoG were quite similar for all the target directions (Fig. 4c,d). Prediction-derived latent dynamics exhibited strong and moderate correlations with the ground-truth-based counterparts for neural mode 1 (0.91) and mode $2(0.50)$, respectively (Fig. 4e). Temporal correlation showed the instantaneous performance, allowing us to identify the time-dependency of similarity. We noticed a consistently strong correlation for mode 1 and a strong correlation during the first 250 ms for mode 2, where a drop occurred, followed by a bounce back to correlated level (Fig, 4f). Even though, neural mode 2 still exhibited high correlation across the time ( $41 \%$ of the time showed the correlation > 0.4 ). These observations held for inverse-NBGNet. Latent trajectories derived from inverse-NBGNet-inferred LFPs and the ground-truth LFPs were highly correlated (Fig. 4g). Similarly, grand-average latent traces for the first neural mode were almost the same for all the targets (Fig. 4h), and a 
strong and moderate correlation was observed in neural mode 1 (0.89), and 2 (0.57; Fig. 4i). Interestingly, the instantaneous correlation was consistently strong over the time interval $(100 \%$, and $84 \%$ of the time $>0.4$ for neural mode 1, and 2; Fig. 4j). We noted that the stronger correlation was associated with the higher ratio of variance the neural mode explained. Thus, we demonstrated that the NBGNet could capture the latent dynamics.

Performance of linear decoder with NBGNet estimations. To understand the information encoded within neural populations, decoding cortical activity is of particular interest ${ }^{37}$. We wondered how accurately linear decoders trained with the model-inferred neural activities would perform (Fig. 5a-b). We first extracted candidate features from the dataset and picked five of them using Fisher score ${ }^{39}$ (Fig. 5c-d). Linear support vector machine (SVM) ${ }^{40}$ classifiers were then trained with selected features (Fig. 5e) to predict the direction of cursor's movement, where the classification accuracy was evaluated using 5-fold cross-validation.

Candidate features were arranged in descending order based on Fisher score averaging across all the channels (Fig. 5d). SVMs were trained with seven conditions: (1) screw ECoG only, (2) reconstructed screw ECoG only, (3) LFP only, (4) reconstructed LFP only, (5) screw ECoG + LFP, (6) reconstructed screw ECoG + LFP, and (7) screw ECoG + reconstructed LFP. Five features were selected for classifiers $1-4$; while ten features were obtained by having five features picked from individual datasets for classifier 5 - 7. Although the classification accuracy of NBGNet-inferred screw ECoG activity (13\%) appears lower than that of ground-truth screw ECoG (19\%), there was no significant difference ( $p>0.05$; Fig. 5f). Incredibly, the classification performance for the predicted LFPs (25\%) was as good as the classifier using features from real LFPs (26\%; $p=0.73$ ), indicating that NBGNet's inference maintained the discriminant power. Also, there was no significant difference when NBGNet's predictions were included for forward (26\% for LFP; $25 \%$ for LFP + reconstructed screw ECoG) and inverse model (19\% for both screw ECoG and screw ECoG + reconstructed LFP). As expected, the classifier trained with LFP and screw ECoG outperformed the other conditions. Surprisingly, we noted a 0.73 -fold decrease in the accuracy of reconstructed LFP and screw ECoG as compared with reconstructed LFP only, resulting from the selection of suboptimal features. Most of the leading discriminant features were from reconstructed LFP when considered simultaneously (Fig. 5d). Together, we demonstrated that the presented model still maintained the integrity of information represented by the neural activity and thus held great potential to improve decoding capability by incorporating appropriate data preprocessing, feature selection, and decoder.

Stable performance across days. As experiments are often performed across multiple sessions, we tested the stability of NBGNet using the same metrics (Supplementary Fig. 4, 8-11). First, the average RMSE between the NBGNet inference and recorded signals for both forward and inverse model were consistent over weeks to a degree almost indistinguishable from that in Day 1 (Fig. 6a-b). As was the case for RMSE, with a few individual trial exceptions, the forward-and inverse-model beta correlation were stable as well (Fig. 6c-d). In one exceptional case we found an unexpected decrease in correlation of a specific channel (Day 2 for forward model; Day 16 for inverse model) due to the amplitude change of the measurements. Overall, the predicted neural activities were still highly correlated with the empirical recordings (coefficient $=0.55$ and 0.50 for forward and inverse model).

We then tested the stability in phase analysis based on the percentage of predictions located in each zone of scatter plot. Forward-NBGNet-inferred screw ECoG were highly phase-synchronized with the real recordings across different days (Fig. 6e). More predictions were always located in the strong synchrony zone than in the poor one $(+39 \%,+21 \%,+23 \%$, $+12 \%,+23 \%$ for Day $1,2,4,12,16$, respectively). Also, reconstructed LFPs were consistently in sync (Fig. 6f). Although 
smaller portion of good synchrony was observed in Day $2(-4 \%)$ and Day 12 (-10\%), more synchronous predictions were inferred across sessions (+4\%/session).

The NBGNet maintained the capability of reconstructing latent dynamics during the repeated movement generation for the full length of recordings from the monkey (Fig. 6g,h). The stability held for a range of manifold dimensionalities from 1 to 3. As we found in Day 1 (Fig. 3e,i), descending trend in the correlations of neural modes was observed for multiple days. The average temporal correlations also showed similar results for both forward and inverse model. We then tested whether NBGNet inferences could be used to predict behavior in different sessions. It was noted that the classifiers performed as well as that trained in different sessions (Fig. 6i). These results provided evidence that NBGNet-derived signals can be employed to predict behavioral variables with similar accuracy as compared with the ground-truth signals for multiple sessions.

Comparison of NBGNet and well-known algorithms. We compared the NBGNet with two conventional alternative approaches, specifically a sphere head mode ${ }^{22,41}$ and RNN (Supplementary Fig. 12-15). Assuming a multi-layered spherical head where each layer represents each brain tissue, the sphere head model provides analytical formulas describing the contribution from current sources to EEG potentials, which are further utilized to approximate the inverse computation. We also applied the machine learning technique, RNN, as a purely date-driven alternative. NBGNet outperformed the purely data-driven RNN and electrophysiology-based sphere head model for multiple days (Fig. 6j). As expected, the performance of analytical solution was the worst due to the non-high-density recordings and the unrealistic assumptions (e.g. isotropic conductivity of the medium). The RNN failed to provide accurate predictions due to the model complexity. In this demonstration, NBGNet gave a more accurate inference consistently over multiple days.

\section{Discussion}

The brain consists of a hierarchical system with multiple levels of organization ${ }^{42}$. To gain a better understanding of brain computations, interest in multi-scale interaction among the genetic, cellular, and macroscale levels has been growing recently, inspiring a shift from emphasizing neural communication in individual scales to exploring the potential associations between scales. However, how these scales are interlinked is still an open question. NBGNet addresses an unmet need to capture the implicit relations of multi-scale brain activity. In the present study we have shown that the neural activity at one scale can be inferred from one another and the performance remains stable over multiple days. The underlying dynamics embedded in NBGNet illustrate how the activity at one scale communicate with other scales, which is a key factor in uncovering the mechanistic understanding of brain computations and the mediation of the behaviors.

To the best of our knowledge, this is the first demonstration of a BG applied to model the multi-scale neural activity. Assuming the brain computation as a nonlinear operator, we employ the deep learning technique to approximate the nonlinear mapping in the BG-derived dynamical systems which describe the translations between different scales. Although channel selection is based on the spatial relationships, NBGNet's performance is not dependent upon the depth (Supplementary Fig. 16), but on the regions (Fig. 2). Forward-NBGNet captures the internal dynamics for performing a center-out task and thus accurately reconstructs the task-related neural activity in premotor, prefrontal cortex, and primary motor cortex. Similarly, inverse-NBGNet-inferred activity matches the ground truth at motor area other than the primary somatosensory cortex. As inverse model is developed by nonlinearizing the inversion of linear forward mapping rather than the direct inversion of nonlinear forward mapping, a slightly poorer performance is observed at more ventral brain region. Since the channel-to-channel communication is modeled individually, scaling up to more channels is expected to 
mitigate the unexpected mismatch. Evidenced with the failure of capturing the noise from unstable recordings, dynamics embedded in the NBGNet are useful for disambiguating brain computations.

The guiding factor in model evaluation is utilizing comprehensive metrics. This is especially important for neuroscientific research. RMSE (Fig. 1) and R-squared (Fig. 6) are used to indicate the absolute and relative measure of fit. Because correlation and coherence are the measures of similarity analyzed in time- and phase-domain, we also employ cross-correlation (Fig. 2) and phase synchrony (Fig. 3) for assessment. Capability of reconstructing the low-dimensional latent dynamics is essential as it holds a key to the understanding of neural mechanisms (Fig. 4). Additionally, we consider the decoding accuracy as an indicator for the applicability of brain-machine interface (Fig. 5). Finally, the predictive power without retraining the model over a long period has recently drawn much attention for the field of neural engineering (Fig. 6). With these comprehensive metrics, we have validated the NBGNet as a reliable approach.

The bias-variance tradeoff is a critical problem in statistics and machine learning, where the simple models have a lower variance yet a higher bias, and the complexity of the model can reduce the bias but increase the variance. It is thus expected that the NBGNet outperforms the analytical sphere head model and the RNN. With the assumptions of the signal sources and the conductivity of the brain tissues, the sphere head model provides a simple solution, but it leads to a large bias error. The data-driven RNN enables the approximation of nonlinear dynamics; however, a large variance, or the socalled "overfitting," can be observed. Combining both electrophysiological modeling and deep learning technique, NBGNet, with the complexity lying between sphere head model and RNN, can capture the patterns in the training data and adapt itself to unseen data, holding a great potential to resolving the bias-variance dilemma.

NBGNet will be helpful in investigating the underlying dynamics in multi-scale brain networks. Modeling the neural activity at disparate scales yields the causal interactions among multiple levels, which is crucial in illuminating the mechanistic understanding of brain computation. Whereas current work focuses on cross-scale interaction, within-scale communication can be incorporated for comprehensive modeling. Additionally, NBGNet can potentially improve the applicability of brain-machine interfaces by inferring the brain activity with increased signal-to-noise ratio. Moreover, the inverse computation to reconstruct the activity at the uncovered brain regions makes LFP-derived whole-brain dynamics available. Taken together, our work represents an important step forward towards the mechanistic modeling of multi-scale neural activity, which may facilitate our understanding of neuropathological activity and the development of clinical devices and rehabilitative therapies to treat abnormal neural activity underlying dysfunctional behaviors.

\section{Acknowledgements}

We thank José del R. Millán from Clinical Neuroprosthetics and Brain Interaction (CNBI) lab at University of Texas at Austin for extensive discussion and suggestions. This research was partially funded by the Defense Advance Research Project Agency (DARPA) under cooperative agreement W911NF-14-2-0043 issued by the Army Research Office contracting office in support of DARPA's SUBNETS program (to J.M.C.). H.-C.Y. is supported in part by National Institutes of Health (GM129617) and Robert A. Welch Foundation (F-1833). Y.-I.C. is supported by the University Graduate Continuing Fellowship at UT Austin.

\section{Contributions}

Y.-J.C. and S.R.S. conceived the project, performed data analysis, and wrote the manuscript with input from all of the authors. J.M.C. and S.R.S. designed and performed the experiments. Y.-J.C. and Y.-I.C. developed the algorithmic approach. Y.-I.C., H.-C.Y., J.M.C., and S.R.S. advised data analysis. 
bioRxiv preprint doi: https://doi.org/10.1101/2020.1130.404244; this version posted December 2, 2020. The copyright holder for this preprint (which was not certified by peer review) is the author/funder, who has granted bioRxiv a license to display the preprint in perpetuity. It is made available under aCC-BY-NC-ND 4.0 International license.

\section{Competing interests}

The authors declare no competing interests.

\section{Corresponding author}

*E-mail: s.santacruz@austin.utexas.edu 


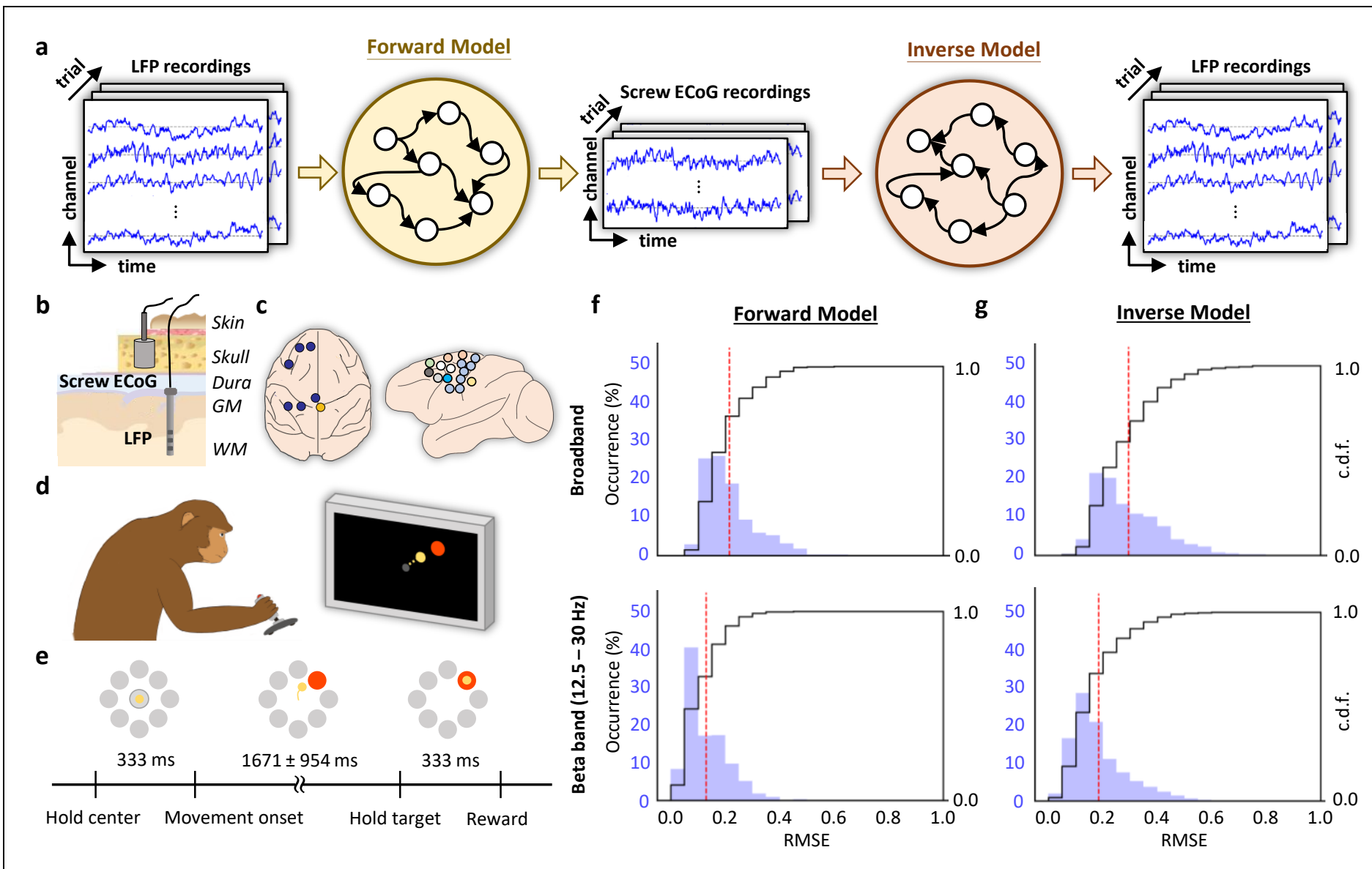

Fig. 1 | NBGNet is a sparse recurrent neural network that utilizes nonlinear dynamics to model the translation between multimodal brain activities. a, Schematic overview of NBGNet architecture for forward and inverse modeling between LFP and screw ECoG. Details are provided in the main text. $\mathbf{b}$, Spatial relationships between LFP and screw ECoG. c, LFP data was acquired from one hemisphere, and screw ECoG signals were recorded across both hemispheres. $\mathbf{d}$, Monkey performed a center-out reaching task using a joystick. e, Schematic of protocol for the experiments. $\mathbf{f , g}$, Histogram and cumulative distribution function (c.d.f.) of RMSE in broadband and beta band $(12.5-30 \mathrm{~Hz})$ for forward model (f) and inverse model ( $\mathbf{g}$; red dashed line: mean). 


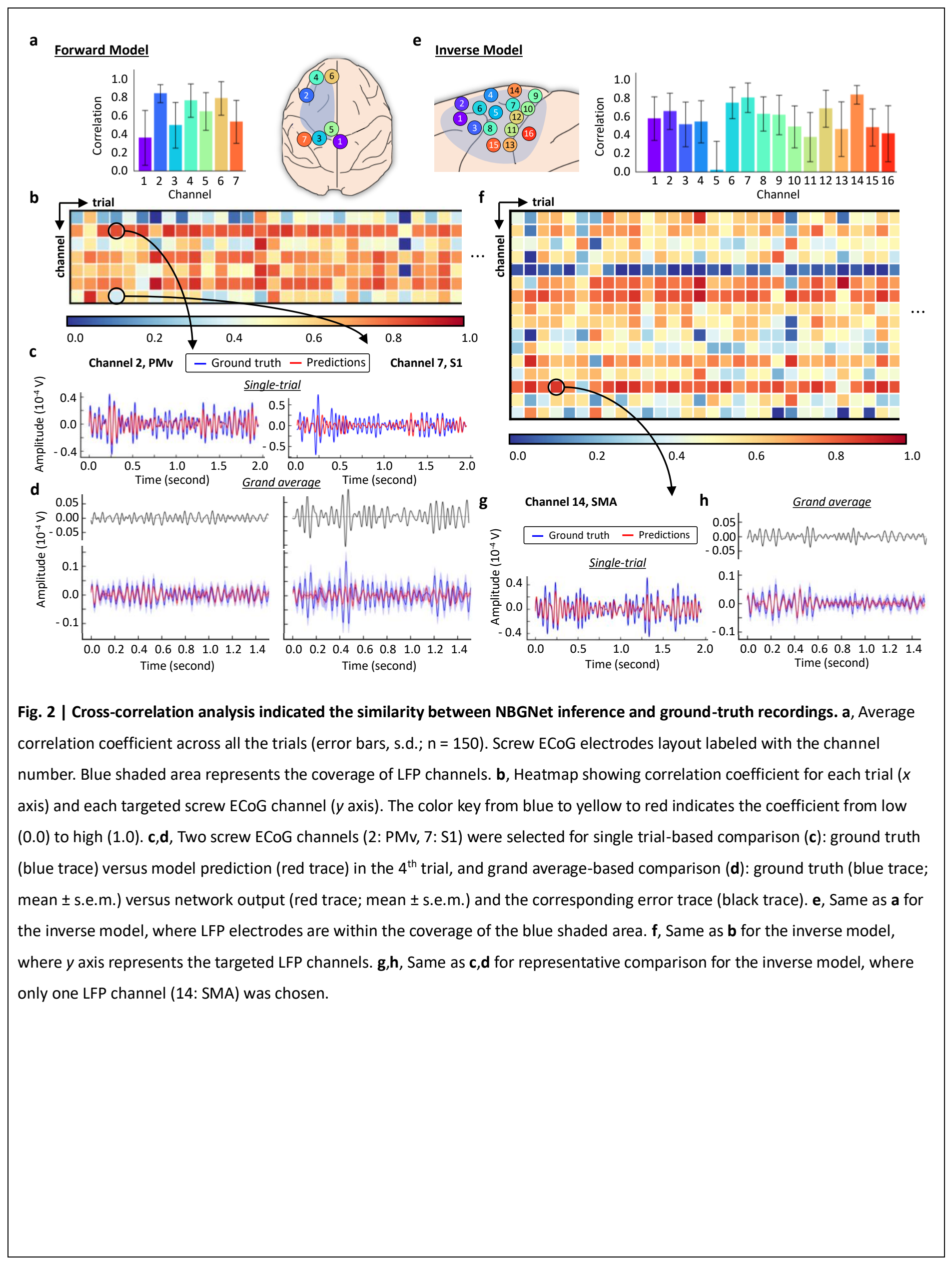



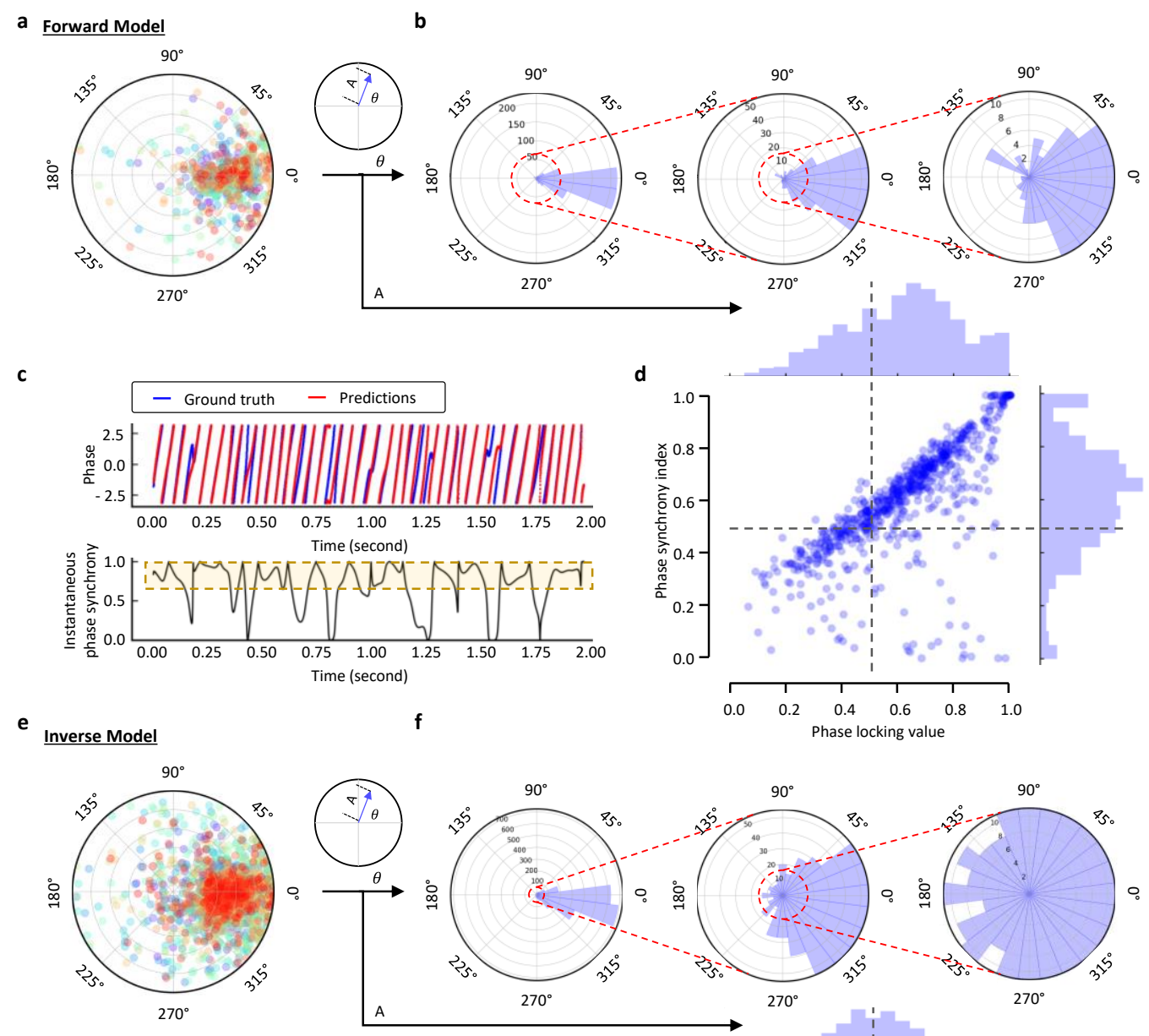

g
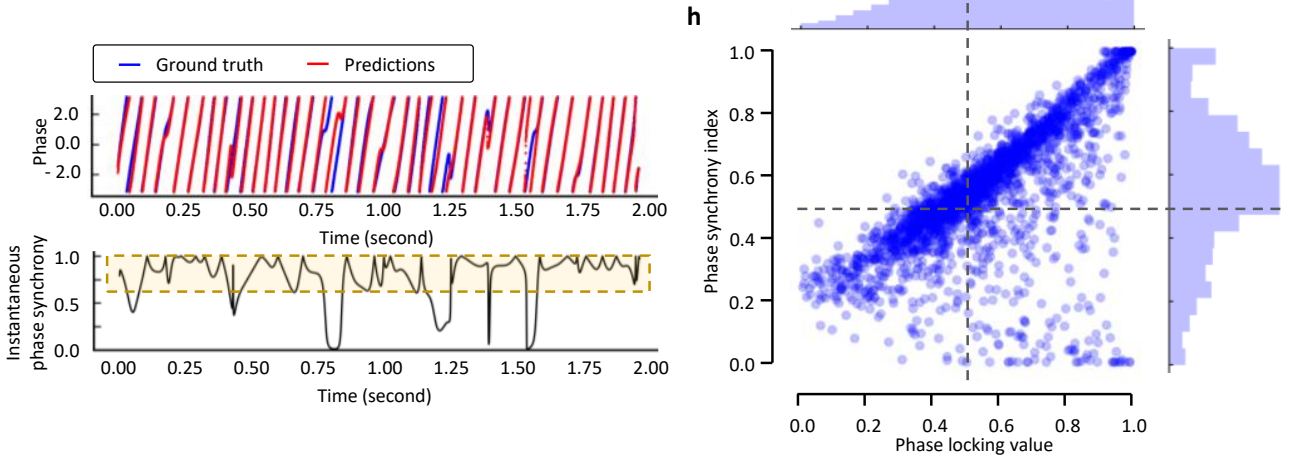

Fig. 3 | Strong phase synchrony between NBGNet estimations and the experimental recordings. a, Polar plots of the mean phase difference averaging across time in each trial for the forward model $(n=1,050)$. $b$, Angular and corresponding zoom-in histogram of the phase of phase-locking derived from (a). c, A screw ECoG channel (2: PMv) was selected for demonstrating that NBGNet made predictions in sync with the ground truth in the $4^{\text {th }}$ trial. Instantaneous phase of the ground truth (blue trace) and the model inference (red trace) at each timepoint (upper) was employed to obtain the instantaneous phase synchrony (lower; black trace) across the time. Yellow area showed a strong synchronization utilized to compute the phase synchrony index (PSI). $\mathbf{d}$, A scatter plot of phase analysis on each channel and each trial, respectively $(n=1,050)$, revealing the expected and hidden relations between PSI and phase-locking value (PLV). Histograms of both PLV and PSI are represented on the $x$ and $y$ axes, respectively. 0.5 was set as thresholds for both PSI and PLV (black dashed line) to identify strong, medium, and poor synchrony regions (Supplementary Fig. 6). e, Same as a for the inverse model $(n=2,400)$. $\mathbf{f}$, Same as $\mathbf{b}$ for the inverse model, where the histogram was derived from e. $\mathbf{g}$, Same

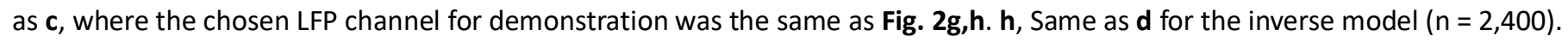



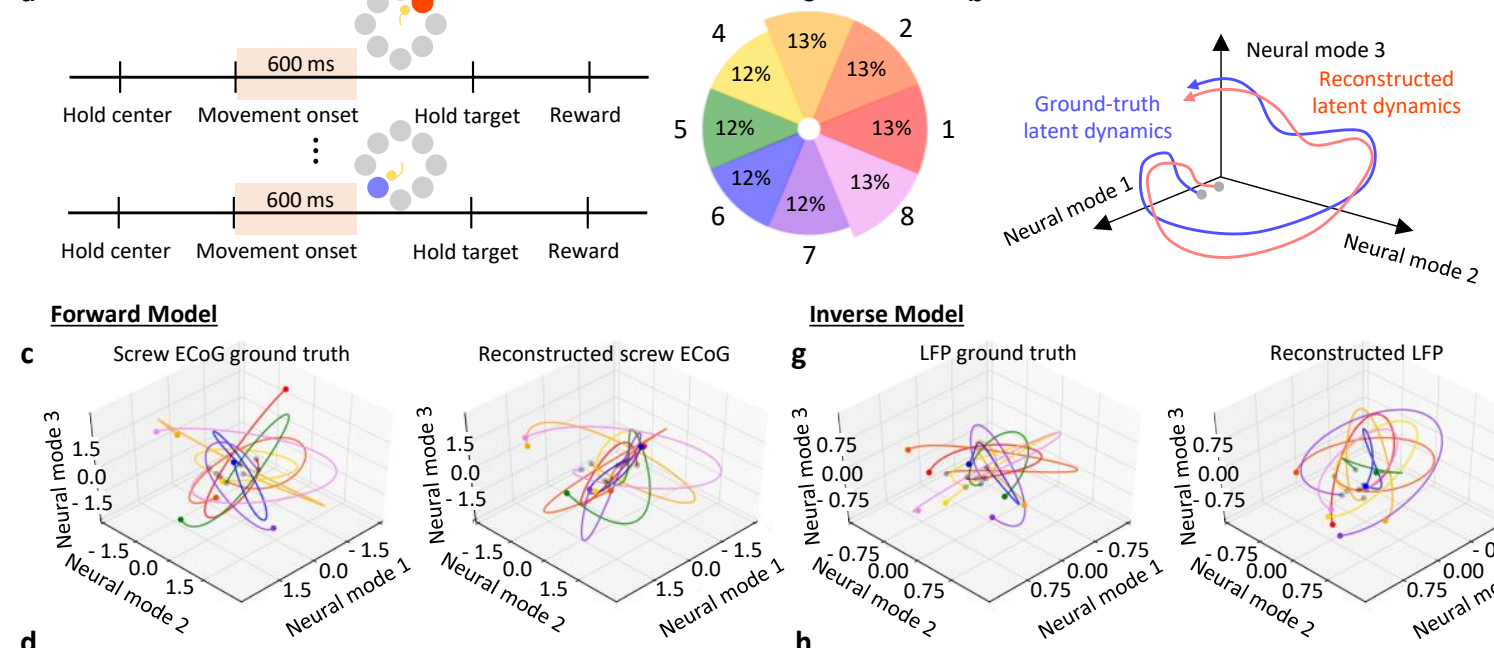

Inverse Model

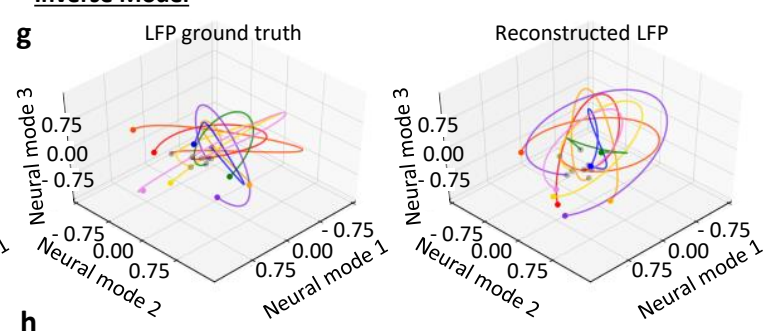

0.0 -1.0

h
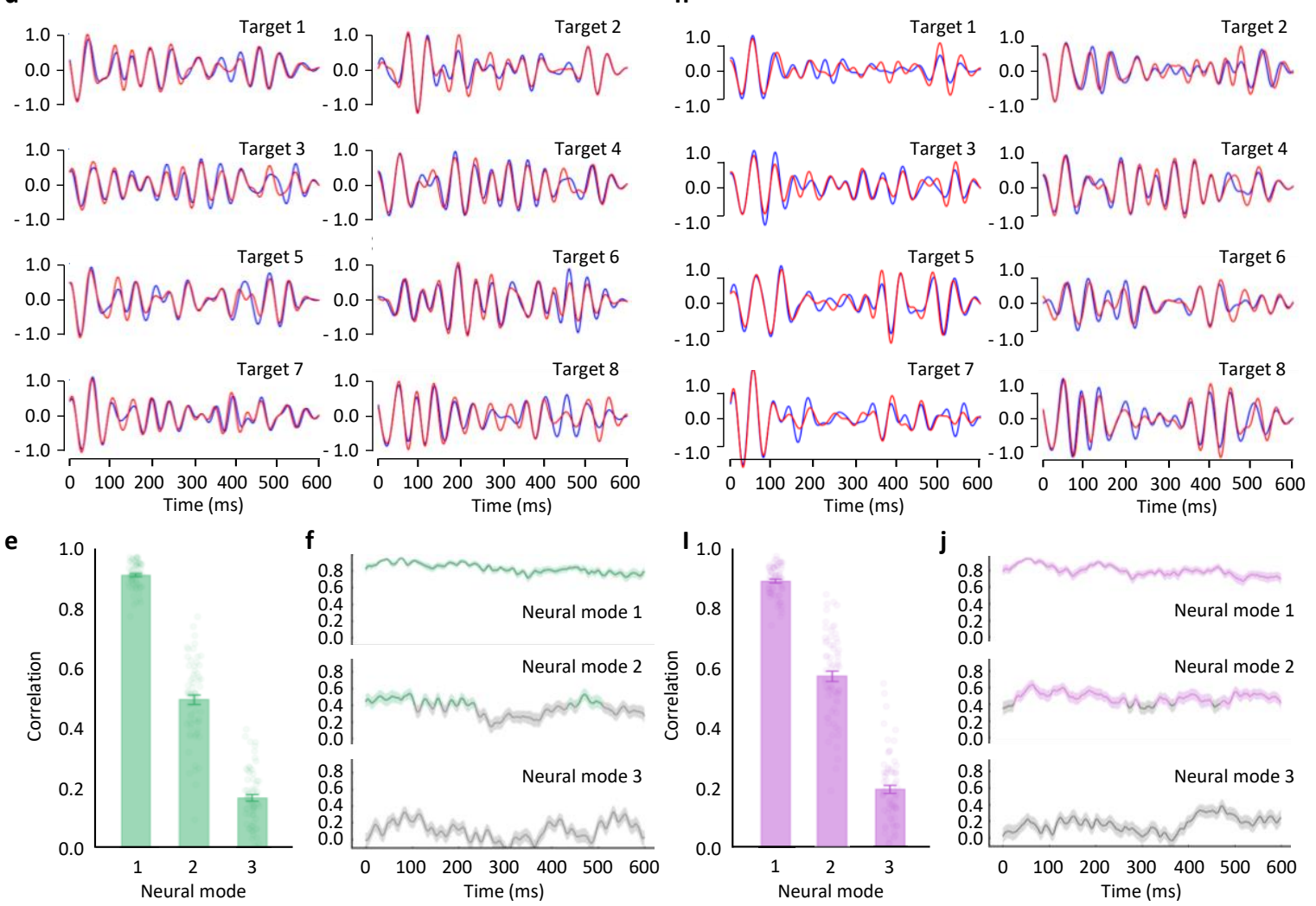

Fig. 4 | NBGNet captures and reconstructs the latent dynamics in the reaching-out task. a, Schematic of protocol indicates the time window used for analysis. Probability of each target direction is uniform. $\mathbf{b}$, We predicted that the latent dynamics can be recovered. c, Representative latent trajectories derived from the ground-truth screw ECoG (left) and reconstructed screw ECoG (right). Each color represents each target direction in a. d, Projection of average ground-truth (blue trace) and reconstructed (red trace) latent trajectories for each target on the first mode. e, Bar plot showing the strong magnitude of the correlations between the ground-truth and reconstructed latent trajectories (error bars, s.e.m.; $n=68$ ). $f$, Temporal correlation trajectories for each neural mode (green trace when above the threshold as 0.4; grey trace as below the threshold; mean \pm s.e.m.). $\mathbf{g}$, Same as $\mathbf{c}$ for the inverse model to reconstruct the latent trajectories derived from LFPs. $\mathbf{h}$, Same as $\mathbf{d}$ for the projection of average ground-truth LFPs-derived (blue trace) and reconstructed LFPs-derived (red trace) latent trajectories. i, Same as e for the correlation between the latent trajectories obtained from recorded LFPs and estimated LFPs. $\mathbf{j}$, Same as for the inverse model (purple trace when above the threshold as 0.4; grey trace as below the threshold). 
bioRxiv preprint doi: https://doi.org/10.1101/2020.11.30.404244; this version posted December 2, 2020. The copyright holder for this preprint (which was not certified by peer review) is the author/funder, who has granted bioRxiv a license to display the preprint in perpetuity. It is made available under aCC-BY-NC-ND 4.0 International license.
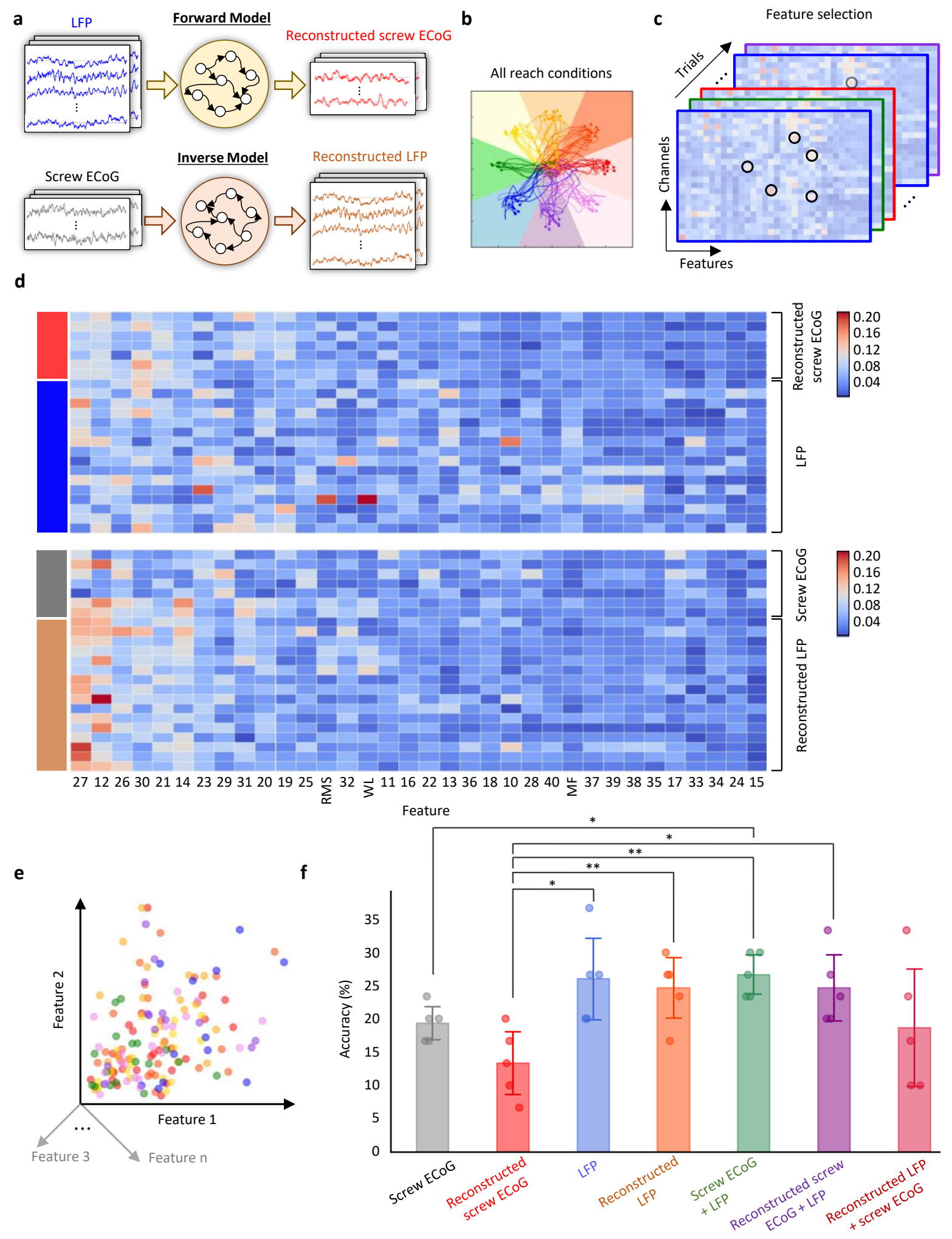

Fig. 5 | NBGNet inference can be used to predict the movement behavior. a, Schematic of NBGNet employed to reconstruct screw ECoG (forward) and LFPs (inverse). b, Individual reaches of a monkey during a center-out task, colored by target location. c, Schematic of feature selection process to select candidates for decoder development. $\mathbf{d}$, Heatmap showing Fisher score for each feature ( $x$ axis) and each channel ( $y$ axis). e, The single-trial features were projected into the feature space, where the linear SVM classifier found the optimal hyperplanes to separate these data points. $f$, Bar plot showing the classification accuracy for each dataset (error bars, s.d.; $n=5$ ). ${ }^{* *} p<0.005, * p<0.05$ using paired T-test. 

available under aCC-BY-NC-ND 4.0 International license.
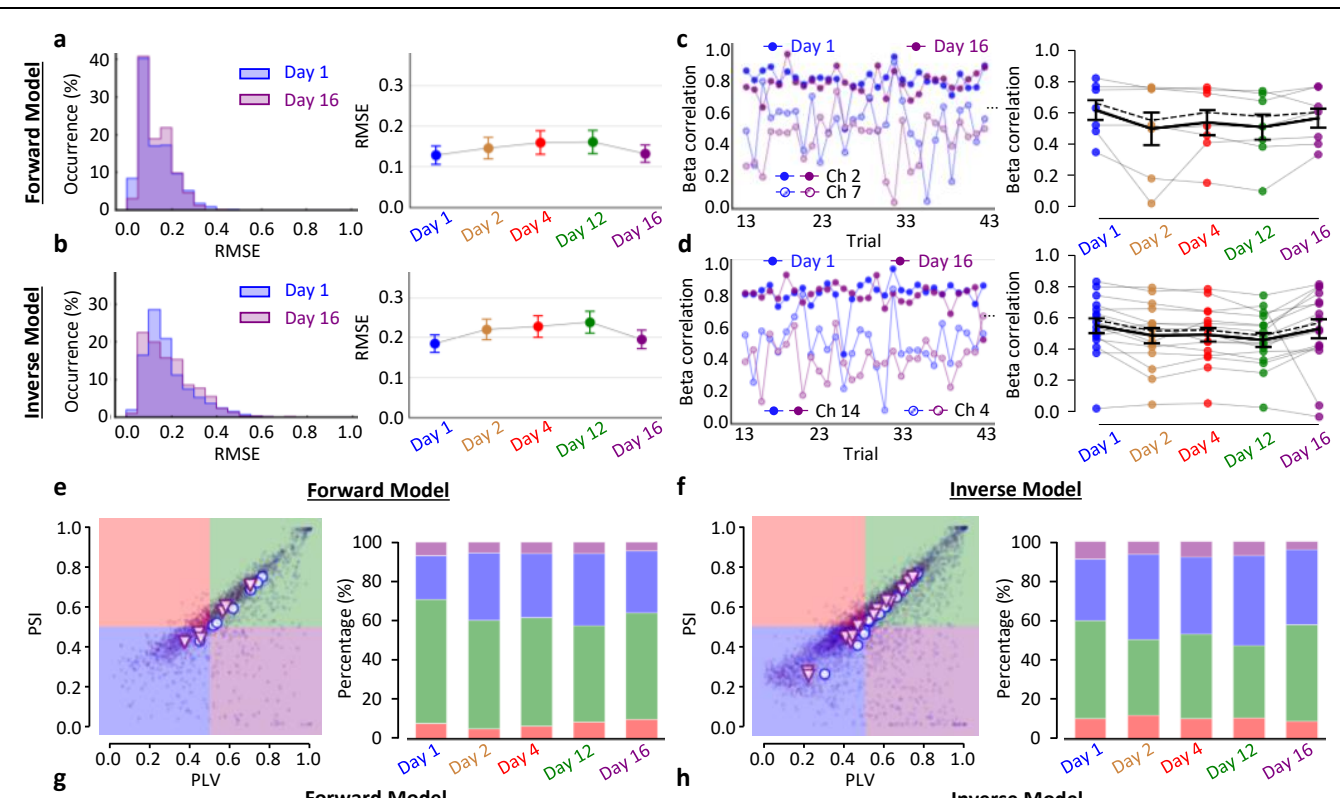

Forward Model

f

Inverse Model
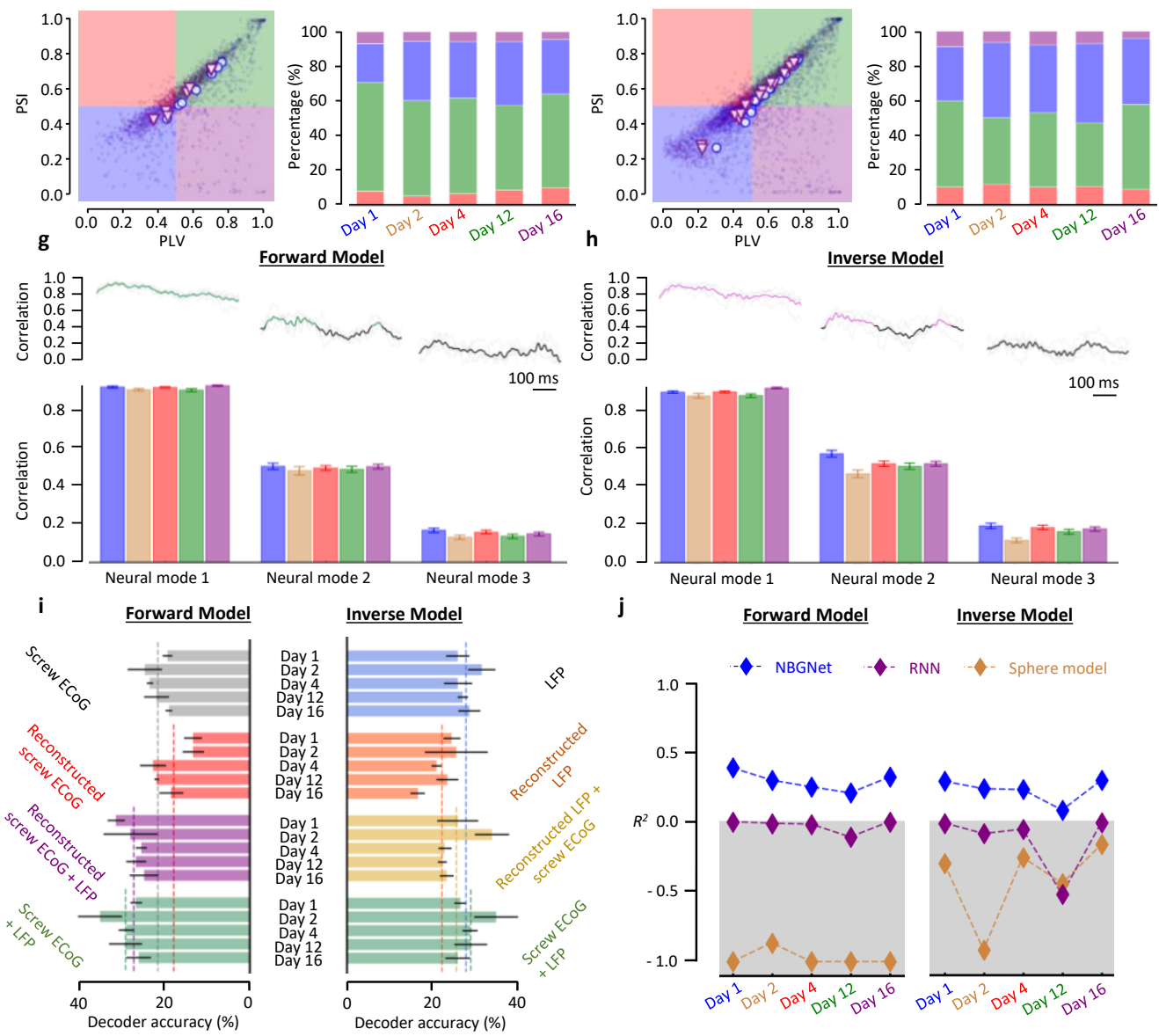

Fig. 6 | Stability of NBGNet's predictions for multiple days. a,b, Histogram of RMSE (left) at Day 1 (blue) and 16 (purple) for the forward (a) and the inverse (b) model. Scatter plot of average RMSE (right) showing no significant difference (error bars, s.e.m.; $n=$ 7 and 16 for $\mathbf{a}$ and b). c,d, Beta correlation (left) at Day 1 (blue) and 16 (purple) for the forward (c) and the inverse (d) model. Scatter plot of average beta correlation, where black solid line is obtained by averaging over the channels and black dashed line left the poorest channel out (error bars, s.e.m.; $\mathbf{n}=7$ and 16 for $\mathbf{c}$ and $\mathbf{d}$ ). e,f, Scatter plot of PSI versus PLI (left) at Day 1 (blue circle) and 16 (purple triangle) for the forward (e) and the inverse (f) model. Stacked bars (right) demonstrate the percentage of predictions locating in each section. $\mathbf{g , h}$, Temporal correlation averaging across days (upper), where colored segments represents stronger correlation as compared with the grey counterparts. Bar plot of average correlation (lower) exhibiting stable performance (error bars; s.e.m.; $n=68,49,128,78,135$ at Day 1, 2, 4, 12, 16). i, Bar plots showing the classification accuracy of linear classifier to predict the target direction (error bars, s.d.; $n=5$ ). Dashed line represents the average performance across days. $\mathbf{j}$, Performance was quantified by measuring variance explained $\left(R^{2}\right)$ between the ground-truth and inferred signals. Dotted lines connect the mean $R^{2}$ value for each population size. 


\section{Reference}

1. Churchland, M. M., Santhanam, G. \& Shenoy, K. V. Preparatory Activity in Premotor and Motor Cortex Reflects the Speed of the Upcoming Reach. Journal of Neurophysiology 96, 3130-3146 (2006).

2. Churchland, M. M., Cunningham, J. P., Kaufman, M. T., Ryu, S. I. \& Shenoy, K. V. Cortical Preparatory Activity: Representation of Movement or First Cog in a Dynamical Machine? Neuron 68, 387-400 (2010).

3. Vyas, S., O’Shea, D. J., Ryu, S. I. \& Shenoy, K. V. Causal Role of Motor Preparation during Error-Driven Learning. Neuron 106, 329-339.e4 (2020).

4. Mauk, M. D. \& Buonomano, D. V. The Neural Basis of Temporal Processing. Annual Review of Neuroscience 27, 307-340 (2004).

5. Remington, E. D., Egger, S. W., Narain, D., Wang, J. \& Jazayeri, M. A Dynamical Systems Perspective on Flexible Motor Timing. Trends in Cognitive Sciences 22, 938-952 (2018).

6. Chaisangmongkon, W., Swaminathan, S. K., Freedman, D. J. \& Wang, X.-J. Computing by Robust Transience: How the Fronto-Parietal Network Performs Sequential, Category-Based Decisions. Neuron 93, 15041517.e4 (2017).

7. Chaudhuri, R. \& Fiete, I. Computational principles of memory. Nature Neuroscience 19, 394-403 (2016).

8. Miller, E. K., Lundqvist, M. \& Bastos, A. M. Working Memory 2.0. Neuron 100, 463-475 (2018).

9. Macke, J. H., Buesing, L. \& Sahani, M. Estimating state and parameters in state space models of spike trains. in Advanced State Space Methods for Neural and Clinical Data (ed. Chen, Z.) 137-159 (Cambridge University Press, 2015). doi:10.1017/СBO9781139941433.007.

10. Byron, M. Y. et al. Gaussian-Process Factor Analysis for Low-Dimensional Single-Trial Analysis of Neural Population Activity. Journal of Neurophysiology 102, 614-635 (2009).

11. Pandarinath, C. et al. Inferring single-trial neural population dynamics using sequential auto-encoders. Nature Methods 15, 805-815 (2018).

12. Buschman, T. J. \& Kastner, S. From behavior to neural dynamics: An integrated theory of attention. Neuron 88, 127-144 (2015).

13. Harbecke, J. The methodological role of mechanistic-computational models in cognitive science. Synthese 1-23 (2020) doi:10.1007/s11229-020-02568-5.

14. Canolty, R. T., Ganguly, K. \& Carmena, J. M. Task-Dependent Changes in Cross-Level Coupling between Single Neurons and Oscillatory Activity in Multiscale Networks. PLOS Computational Biology 8, e1002809 (2012).

15. Michel, C. M. \& Brunet, D. EEG Source Imaging: A Practical Review of the Analysis Steps. Front. Neurol. 10, (2019).

16. Vorwerk, J., Aydin, Ü., Wolters, C. H. \& Butson, C. R. Influence of Head Tissue Conductivity Uncertainties on EEG Dipole Reconstruction. Front. Neurosci. 13, 531 (2019).

17. Fernández, B., Prabhudesai, A. V., Murty, V. V., Gupta, R. \& Chang, W. R. Neurobondgraphs: modeling environment of nonlinear dynamic systems using neural networks and bond graphs. in 75-90 (ASME, 1992).

18. Paynter, H. M. Analysis and design of engineering systems. (MIT Press, 1961).

19. Bond Graphs for Modelling, Control and Fault Diagnosis of Engineering Systems. (Berlin: Springer, 2017). doi:10.1007/978-3-319-47434-2. 
20. Yeager, J. D., Phillips, D. J., Rector, D. M. \& Bahr, D. F. Characterization of flexible ECoG electrode arrays for chronic recording in awake rats. Journal of Neuroscience Methods 173, 279-285 (2008).

21. Choi, H. et al. Long-term evaluation and feasibility study of the insulated screw electrode for ECoG recording. Journal of Neuroscience Methods 308, 261-268 (2018).

22. Grech, R. et al. Review on solving the inverse problem in EEG source analysis. Journal of NeuroEngineering and Rehabilitation 5, 25 (2008).

23. Sanes, J. N. \& Donoghue, J. P. Oscillations in local field potentials of the primate motor cortex during voluntary movement. PNAS 90, 4470-4474 (1993).

24. Khanna, P. \& Carmena, J. M. Beta band oscillations in motor cortex reflect neural population signals that delay movement onset. ELife 6, e24573 (2017).

25. Dancey, C. P. \& Reidy, J. Statistics without maths for psychology. (Pearson education, 2007).

26. Akoglu, H. User's guide to correlation coefficients. Turkish Journal of Emergency Medicine 18, 91-93 (2018).

27. Lachaux, J.-P., Rodriguez, E., Martinerie, J. \& Varela, F. J. Measuring phase synchrony in brain signals. Human Brain Mapping 8, 194-208 (1999).

28. Mormann, F., Lehnertz, K., David, P. \& Elger, C. E. Mean phase coherence as a measure for phase synchronization and its application to the EEG of epilepsy patients. Physica D: Nonlinear Phenomena 144, 358-369 (2000).

29. Afshar, A. et al. Single-Trial Neural Correlates of Arm Movement Preparation. Neuron 71, 555-564 (2011).

30. Churchland, M. M. et al. Neural population dynamics during reaching. Nature 487, 51-56 (2012).

31. Kaufman, M. T., Churchland, M. M., Ryu, S. I. \& Shenoy, K. V. Cortical activity in the null space: permitting preparation without movement. Nature Neuroscience 17, 440-448 (2014).

32. Mante, V., Sussillo, D., Shenoy, K. V. \& Newsome, W. T. Context-dependent computation by recurrent dynamics in prefrontal cortex. Nature 503, 78-84 (2013).

33. Sadtler, P. T. et al. Neural constraints on learning. Nature 512, 423-426 (2014).

34. Wold, S., Esbensen, K. \& Geladi, P. Principal component analysis. Chemometrics and Intelligent Laboratory Systems 2, 37-52 (1987).

35. Gallego, J. A., Perich, M. G., Miller, L. E. \& Solla, S. A. Neural Manifolds for the Control of Movement. Neuron 94, 978-984 (2017).

36. Thompson, B. Canonical Correlation Analysis. in Encyclopedia of Statistics in Behavioral Science (eds. Everitt, B. S. \& Howell, D.) (Wiley, 2005). doi:10.1002/0470013192.bsa068.

37. Gallego, J. A. et al. Cortical population activity within a preserved neural manifold underlies multiple motor behaviors. Nature Communications 9, 1-13 (2018).

38. Winkler, A. M., Renaud, O., Smith, S. M. \& Nichols, T. E. Permutation Inference for Canonical Correlation Analysis. arXiv reprint arXiv:2002.10046 (2020).

39. Duda, R. O., Hart, P. E. \& Stork, D. G. Pattern Classification. (John Wiley \& Sons, 2012).

40. Cortes, C. \& Vapnik, V. Support-vector networks. Mach Learn 20, 273-297 (1995).

41. Hallez, H. et al. Review on solving the forward problem in EEG source analysis. Journal of NeuroEngineering and Rehabilitation 4, 46 (2007).

42. Heuvel, M. P. van den, Scholtens, L. H. \& Kahn, R. S. Multiscale Neuroscience of Psychiatric Disorders. 
bioRxiv preprint doi: https://doi.org/10.1101/2020.11.30.404244; this version posted December 2, 2020. The copyright holder for this preprint (which was not certified by peer review) is the author/funder, who has granted bioRxiv a license to display the preprint in perpetuity. It is made available under aCC-BY-NC-ND 4.0 International license.

\section{Biological Psychiatry 86, 512-522 (2019).}




\section{Methods}

The NBGNet model. The NeuroBondGraph Network. The NBGNet model is a sparse recurrent neural network (RNN) that captures the system dynamics functionality, especially causality. RNNs make use of sequential information, assuming the output being depended on the previous computations. Accordingly, RNNs have the memory to capture the information about what has been calculated so far. Evidenced with the fact that the recurrent connections exist in visual cortex and hippocampus ${ }^{43}$, RNN is crucial to capture the neural dynamics. Unlike a "black box" model, RNNs in NBGNet are sparsely connected, creating a "gray-box" recurrent neural model using a priori knowledge about the system from the Bond Graphs $(B G s)^{18}$. BGs are regarded as powerful tools to model the physical system by investigating how energy is generated, transferred, transformed, and dissipated among the different system components. BGs allow us to obtain the full representation of the dynamics of the system, and then extract the nonlinear dynamic equation describing the system. Starting with modeling the signal translation between brain activity recorded from different modalities, we can obtain the underlying nonlinear dynamic equations and generate the corresponding NBGNet. In the end, the well-trained network served as the solution to inferring the neural activity from one another.

Bond Graphs modeling. Bond graph is a graphical representation of a physical system that allows us to describe the system with a state-space representation. BG consists of the "bonds," representing the power which is categorized into two parts: flow and effort, and the elements, including single-port, and multi-port components. Literally, single-port elements only have one port for the linkage. Such elements include (1) sources, denoted as $S_{e}$ or $S_{f}$, serving as the input effort or flow for the system, (2) sinks, denoted as the same as the sources, serving as the output effort or flow for the system, (3) inertia elements, denoted as $I$, are the ones that store energy, (4) resistance elements, denoted as $R$, are the elements that dissipate energy, and (5) compliance elements, denoted as $C$, are the elements that store potential energy. Furthermore, multi-port elements such as 0 -junctions and 1-junctions are employed to split power across their ports. The properties of 0 -junctions are that all efforts are equal across the bonds and the sum of flow in equals to the sum of flow out. In contrast, 1 -junctions represent that all flows are equal across the bonds and the sum of effort in equals to the sum of effort out. The main concept of BG is to link each component in the graph with the "bonds" which represent the bi-directional exchange of energy. Each bond has two features, half-arrow and causality. Half-arrow is used to provide the sign convention for the work being done. Accordingly, sources will always have the arrow pointing away from the element, while the rest of the single-port elements will have the arrow pointing into the elements. Causality in BG denotes which side of the bond governs the instantaneous effort and flow. The causal stroke is assigned to one end of the bond to represent the side with causality defines the flow while the opposite end defines the effort. In general, since two passive components, $I$ and $C$, exhibit time-dependence behavior, they have preferred causal orientation with $C$ component defining the effort and $I$ component defining the flow. Since BGs are domain neutral, where energy in different domains (e.g., linear mechanical, angular mechanical, electromagnetic, and hydraulic) can be transferred into each other with a constant, this dynamical modeling can incorporate physical systems in multiple domains.

Bond Graphs forward modeling. The interactions between recording systems were modeled based on the physiology of brain tissue and its effect on the electrical signal flow. Screw ECoG signals were recorded within the skull while LFP signals were measured within the cortex. Therefore, the brain tissues between the recording location of screw ECoG and LFP are skull, dura mater, and cortex. Assuming that the LFP recordings are the electrical source, the screw ECoG is the measurement of the voltage, and brain tissues represented the effective impedance, we can model it as an electrical circuit. 
The skull is the bony structure which contains sinus cavities and numerous foramina. From the anatomy, the skull consists of three layers: a spongy bone layer in the middle of two compact bone layers. The cavities in the spongy bone can be modeled as a capacitance that provides a potential inside them. The compact bone and the trabeculae of the spongy bone can be modeled as resistances. Various potential paths for electrical signals to travel are taken into account to construct a simplified electrical model to study the signal propagation. As dura mater is a thick membrane surrounding the brain, dura model is built with the effective resistance and capacitance in parallel. Although cortex is composed of folded grey matter, it is modeled as an effective resistance to simplify the complexity. Combing together, LFP-screw ECoG transmission electrical circuit can be established (Supplementary Fig. 1a). With the effective electrical circuit for electrical signal pathways, Bond Graph is then generated (Supplementary Fig. 1b), where the charges, $q$, are represented the states of the physical systems. Given the causality assignment, the system is $3^{\text {rd }}$ order. The ordinary differential equations for the system are derived as follows:

$$
\begin{aligned}
& \dot{q}_{1}=\dot{q}_{2}+\frac{q_{2}}{R_{2} C_{2}}+\frac{1}{R_{5}}\left(\frac{q_{2}}{C_{2}}-\frac{q_{3}}{C_{3}}\right)-\frac{q_{1}}{R_{1} C_{1}} \\
& \dot{q}_{2}=\frac{1}{1+\frac{R_{0}+R_{\text {ScrewECoG }}}{R_{4}}}\left(\frac{1}{R_{4}}\left(V_{L F P}-\frac{q_{1}}{C_{1}}-\frac{q_{2}}{C_{2}}-\left(R_{0}+R_{\text {ScrewECoG }}\right)\left(\frac{q_{2}}{R_{2} C_{2}}+\frac{1}{R_{5}}\left(\frac{q_{2}}{C_{2}}-\frac{q_{3}}{C_{3}}\right)\right)\right)-\frac{q_{2}}{R_{2} C_{2}}-\frac{1}{R_{5}}\left(\frac{q_{2}}{C_{2}}-\frac{q_{3}}{C_{3}}\right)\right) \\
& \dot{q}_{3}=\frac{1}{R_{5}}\left(\frac{q_{2}}{C_{2}}-\frac{q_{3}}{C_{3}}\right)-\frac{q_{3}}{R_{3} C_{3}} \\
& V_{\text {ScrewECoG }}=R_{\text {ScrewECoG }}\left(\frac{1}{R_{0}+R_{4}+R_{\text {ScrewECoG }}}\left(V_{L F P}-\frac{q_{1}}{C_{1}}-\frac{q_{2}}{C_{2}}\right)\right)
\end{aligned}
$$

While the equations above represent the ideal condition where the resistance $R$ and the capacitance $C$ are linear. Considering the uncertainty and continuous changing of human's brain tissue, nonlinearity is introduced in the equation:

$$
\begin{gathered}
\dot{q}_{1}=F_{R_{T}}^{-1}\left(u-F_{C_{1}}^{-1}\left(q_{1}\right)-F_{C_{2}}^{-1}\left(q_{2}\right)\right)-F_{R_{1}}^{-1}\left(F_{C_{1}}^{-1}\left(q_{1}\right)\right) \\
\dot{q}_{2}=F_{R_{T}}^{-1}\left(u-F_{C_{1}}^{-1}\left(q_{1}\right)-F_{C_{2}}^{-1}\left(q_{2}\right)\right)-F_{R_{2}}^{-1}\left(F_{C_{2}}^{-1}\left(q_{2}\right)\right)-F_{R_{5}}^{-1}\left(F_{C_{2}}^{-1}\left(q_{2}\right)-F_{C_{3}}^{-1}\left(q_{3}\right)\right) \\
\dot{q}_{3}=F_{R_{5}}^{-1}\left(F_{C_{2}}^{-1}\left(q_{2}\right)-F_{C_{3}}^{-1}\left(q_{3}\right)\right)-F_{R_{3}}^{-1}\left(F_{C_{3}}^{-1}\left(q_{3}\right)\right) \\
y=F_{R_{\text {screwECoG }}\left(F_{R_{T}}^{-1}\left(u-F_{C_{1}}^{-1}\left(q_{1}\right)-F_{C_{2}}^{-1}\left(q_{2}\right)\right)\right)}
\end{gathered}
$$

where, $R_{T}$ represents $R_{0}+R_{4}+R_{\text {ScrewECoG }}, u$ represents $V_{L F P}, y$ represents $V_{\text {ScrewECoG }}$, and $F(\cdot)$ is a nonlinear function to be determined.

Bond Graphs inverse modeling. The multi-variable time varying Bond Graph forward model, equation (1-2), can be expressed as the state-space representation,

$$
\begin{aligned}
& \dot{x}=\mathbf{A} x+\mathbf{B} u \\
& y=\mathbf{C} x+\mathbf{D} u
\end{aligned}
$$

where, $x=\left[q_{1}, q_{2}, q_{3}\right]^{T}, u=V_{L F P}, y=V_{\text {screwECoG }}$,

$$
\mathbf{A}=\left[\begin{array}{ccc}
\left(\frac{-1}{R_{\text {screwECoG }}+R_{0}+R_{4}}+\frac{-1}{R_{1}}\right) \frac{1}{C_{1}} & \left(\frac{-1}{R_{\text {screwECoG }}+R_{0}+R_{4}}\right) \frac{1}{C_{2}} & 0 \\
\left(\frac{-1}{R_{\text {screwECoG }}+R_{0}+R_{4}}\right) \frac{1}{C_{1}} & \left(\frac{-1}{R_{\text {screwECoG }}+R_{0}+R_{4}}+\frac{-1}{R_{2}}+\frac{-1}{R_{5}}\right) \frac{1}{C_{2}} & \frac{1}{R_{5} C_{3}} \\
0 & \frac{1}{R_{5} C_{2}} & \left(\frac{-1}{R_{5}}+\frac{-1}{R_{3}}\right) \frac{1}{C_{3}}
\end{array}\right]
$$




$$
\begin{aligned}
& \mathbf{B}=\left[\begin{array}{lll}
\frac{1}{R_{\text {ScrewECoG }}+R_{0}+R_{4}} & \frac{1}{R_{\text {ScrewECoG }}+R_{0}+R_{4}} & 0
\end{array}\right]^{T} \\
& \mathbf{C}=\left[\begin{array}{lll}
\frac{-R_{\text {ScrewECoG }}}{\left(R_{\text {ScrewECoG }}+R_{0}+R_{4}\right) C_{1}} & \frac{-R_{\text {ScrewECoG }}}{\left(R_{\text {ScrewECoG }}+R_{0}+R_{4}\right) C_{2}} & 0
\end{array}\right] \\
& \mathbf{D}=\left[\frac{R_{\text {ScrewECoG }}}{R_{\text {ScrewECoG }}+R_{0}+R_{4}}\right]
\end{aligned}
$$

The inversion algorithm for multi-variable system were obtained by Sain and Massey ${ }^{44}$,

$$
\begin{gathered}
\dot{q}_{1}=-\frac{q_{1}}{R_{1} C_{1}}+\frac{V_{\text {screwECoG }}}{R_{\text {ScrewECoG }}} \\
\dot{q}_{2}=-\frac{q_{2}}{R_{2} C_{2}}-\frac{1}{R_{5}}\left(\frac{q_{2}}{C_{2}}-\frac{q_{3}}{C_{3}}\right)+\frac{V_{\text {ScrewECoG }}}{R_{\text {ScrewECoG }}} \\
\dot{q}_{3}=\frac{1}{R_{5}}\left(\frac{q_{2}}{C_{2}}-\frac{q_{3}}{C_{3}}\right)-\frac{q_{3}}{R_{3} C_{3}} \\
V_{L F P}=\frac{q_{1}}{C_{1}}+\frac{q_{2}}{C_{2}}+\frac{R_{\text {ScrewECoG }}+R_{0}+R_{4}}{R_{\text {ScrewECoG }}} V_{\text {ScrewECoG }}
\end{gathered}
$$

As forward model, nonlinearity is introduced in the equation as well,

$$
\begin{gathered}
\dot{q}_{1}=F_{R_{\text {screwECoG }}^{-1}}(y)-F_{R_{1}}^{-1}\left(F_{C_{1}}^{-1}\left(q_{1}\right)\right) \\
\dot{q}_{2}=F_{R_{\text {screwECoG }}}^{-1}(y)-F_{R_{2}}^{-1}\left(F_{C_{2}}^{-1}\left(q_{2}\right)\right)-F_{R_{5}}^{-1}\left(F_{C_{2}}^{-1}\left(q_{2}\right)-F_{C_{3}}^{-1}\left(q_{3}\right)\right) \\
\dot{q}_{3}=F_{R_{5}}^{-1}\left(F_{C_{2}}^{-1}\left(q_{2}\right)-F_{C_{3}}^{-1}\left(q_{3}\right)\right)-F_{R_{3}}^{-1}\left(F_{C_{3}}^{-1}\left(q_{3}\right)\right) \\
u=F_{C_{1}}^{-1}\left(q_{1}\right)+F_{C_{2}}^{-1}\left(q_{2}\right)+F_{R_{T}}\left(F_{R_{\text {screwECoG }}^{-1}}(y)\right)
\end{gathered}
$$

where, $R_{T}$ represents $R_{0}+R_{4}+R_{\text {ScrewECoG }}, u$ represents $V_{L F P}, y$ represents $V_{\text {ScrewECoG }}$, and $F(\cdot)$ is a nonlinear function to be determined.

NeuroBondGraphs Network modeling. To approximate the unknown nonlinear relationship, artificial neural network technique is introduced to incorporate the knowledge obtained from the pattern recognition capabilities of neural network and the physical information about the system via BG. Each nonlinear function is approximated through a multi-layer perceptron (MLP) unit. The architecture of NBGNet is a sparse recurrent MLP network which can identify the nonlinear dynamics recursively.

The full NBGNet inference model. The full NBGNet, both forward and inverse models, are run in the following way. First, a data trial is chosen. Then, for each time step from 1 to $T$, the network is updated and eventually predicts the output according to

$$
\begin{gathered}
\mathbf{V}_{\text {screwECoG }, t}, \mathbf{q}_{t+1}^{\text {forward }}=\mathrm{NBG}^{\text {forward }}\left(\mathbf{q}_{t}^{\text {forward }}, \mathbf{V}_{L F P, t}\right) \\
\mathbf{V}_{L F P, t}, \mathbf{q}_{t+1}^{\text {inverse }}=\mathrm{NBG}^{\text {inverse }}\left(\mathbf{q}_{t}^{\text {inverse }}, \mathbf{V}_{\text {screwECoG }, t}\right)
\end{gathered}
$$


After training, the full model can be run, starting with any single trial or a set of trials corresponding to the particular experimental conditions, to obtain the associated dynamic states, and inferred target signals for that trial or condition.

The loss function. To optimize our model, we would like to minimize the mean-squared-error (MSE) of the data, which measures the average of the squares of the errors. Given the ground-truth measurement $\mathbf{Y}_{g t}$ and the model prediction $\mathbf{Y}_{\text {pre }}$, the MSE is calculated as follows,

$$
\operatorname{MSE}=\frac{1}{T} \sum_{t=0}^{T-1}\left[\mathbf{Y}_{g t}(t)-\mathbf{Y}_{\text {pre }}(t)\right]^{2}
$$

where, $T$ is the number of time points in the given trial. The loss function will be minimized by backpropagating the error and update the parameters of the model iteratively.

Hyperparameters and further details of NBGNet implementation. The major hyperparameters for forward and inverse model is the number of hidden nodes in the MLP unit for nonlinear mapping estimation and the time step. For both forward and inverse model, 7 nodes were utilized in MLP units, and the time step of NBGNet was equal to the data bin size. To help avoid over-fitting, we selected different trials of data for model training when NBGNet has been updated for twenty times. Furthermore, NBGNet is sparsely connected rather than fully connected, which reduces the complexity of the model.

NBGNets were randomly initialized by Glorot uniform initializer and optimized using adaptive moment estimation (Adam) optimizer with a starting learning rate of $1 \times 10^{-3}$. The framework was implemented with TensorFlow version 1.15 .0 and Python version 3.7.4 in the Microsoft Windows 10 operating system. The training was performed on a consumer-built desktop equipped with 4 GPUs (Gigabyte RTX 2080 Ti graphics cards, NVIDIA) and a Core i9-9820X CPU @ 3.3 GHz (Intel). To monitor overfitting, a portion of the data is set aside as the validation set, and these data are not used to update the weights of NBGNet. Instead, they are used to evaluate the predictive performance on held-out data. Here we used a ratio of 9:1 between training and validation data.

Behavioral tasks. The macaque monkey was trained in a center-out task. Briefly, the subject was trained to use a joystick to move a cursor on a computer screen from a center target to a peripheral target. The joystick was located in front of the primate chair and the subject was free to use either hand to control the joystick during the experiment. In the task, the subject was trained to fold the cursor at the center target for $320 \mathrm{~ms}$. Then the subject was presented with one of the eight outer targets, equally spaced in a circle and selected randomly with uniform probability. The subject moved the cursor to the peripheral target and held the cursor inside the target for $320 \mathrm{~ms}$. A trial was considered to be successful if the subject completed the 320 ms hold-center followed by holding at the peripheral target for 320 ms. The reward was scheduled after a successful trial, where a custom-programmed Arduino triggered the reward system to deliver a small amount of juice to the subject.

Scale-dependent analysis. To evaluate how close the model predictions are to the ground-truth signals, root mean square error (RMSE) is commonly used to indicate the absolute fit of the model. RMSE is defined as the square root of the mean of the square of the error,

$$
\operatorname{RMSE}=\sqrt{\frac{1}{T} \sum_{t=1}^{T}\left[\mathbf{Y}_{g t}(t)-\mathbf{Y}_{p r e}(t)\right]^{2}}
$$


where the $\mathbf{Y}_{g t}$ represents ground-truth measurement, $\mathbf{Y}_{\text {pre }}$ represents the model prediction, and $T$ is the number of time points in the given trial.

Similarity analysis. Similarity of two time series signals also conveys an important message whether two time series signals exhibit similar shape of oscillation. Here we use Pearson correlation coefficient to measure how highly correlated two time series signals are.

$$
\rho\left(\mathbf{Y}_{g t}, \mathbf{Y}_{\text {pre }}\right)=\frac{\sum \mathbf{Y}_{g t} \mathbf{Y}_{\text {pre }}-\frac{\sum \mathbf{Y}_{g t} \sum \mathbf{Y}_{\text {pre }}}{T}}{\sqrt{\left(\sum \mathbf{Y}_{g t}^{2}-\frac{\left(\sum \mathbf{Y}_{g t}\right)^{2}}{T}\right)\left(\sum \mathbf{Y}_{\text {pre }}^{2}-\frac{\left(\sum \mathbf{Y}_{p r e}\right)^{2}}{T}\right)}}
$$

where the $\mathbf{Y}_{g t}$ represents ground-truth measurement, $\mathbf{Y}_{\text {pre }}$ represents the model prediction, and $T$ is the number of time points in the given trial.

Phase analysis. On top of the time-domain, phase-domain reveals other characteristics that are not visible in time-domain. Especially in neural engineering field, the synchronization of the neural activity is one of the properties that can only be quantified in phase domain. Given a pair of signals, $\mathbf{s}_{1}(t)$ and $\mathbf{s}_{2}(t)$, which have been band-pass filtered to a frequency range of interest, Hilbert transform, $\mathbf{H T}[\cdot]$, will be applied to obtain the corresponding analytical signals, $\mathbf{z}_{1}(t)$ and $\mathbf{z}_{2}(t)$,

$$
\begin{aligned}
\mathbf{z}_{i}(t) & =\mathbf{s}_{i}(t)+j \mathbf{H T}\left[\mathbf{s}_{i}(t)\right]=\mathbf{A}_{i}(t) e^{j \boldsymbol{\phi}_{i}(t)} \\
\mathbf{H T}\left[\mathbf{s}_{i}\left(t_{k}\right)\right] & =\mathbf{s}_{i}\left(t_{k}\right) * \frac{1}{2 \pi}\left[\int_{-\pi}^{0} j \cdot e^{j w k} d w-\int_{0}^{\pi} j \cdot e^{j w k} d w\right]
\end{aligned}
$$

where $k=1$ to $T, \mathbf{A}_{i}(t)$ represents the instantaneous amplitude, and $\boldsymbol{\phi}_{i}(t)$ represents the instantaneous phase. In order to obtain a comprehensive view, we utilized two metrics: phase-locking value and phase synchrony index. Phase locking value ${ }^{27}, P L V$, or so-called mean phase coherence ${ }^{28}$, is defined as,

$$
P L V=\left|\frac{1}{T} \sum_{i=0}^{T-1} e^{j(\Delta \boldsymbol{\phi}(t))}\right|
$$

It is utilized to measure the intra-trial variability of the phase difference between two signals. In addition, the phase of phase-locking can be extracted to evaluate the mean phase different across time.

On top of $P L V$, we are also interested in the instantaneous performance, and thus we introduce phase synchrony index. First, provided with the instantaneous phase of two time series signals, $\boldsymbol{\phi}_{1}(t)$ and $\boldsymbol{\phi}_{2}(t)$, the instantaneous phase synchrony, IPS, which measures the phase similarity at each timepoint, is calculated by

$$
\operatorname{IPS}(t)=1-\sin \left(\frac{\left|\phi_{1}(t)-\phi_{2}(t)\right|}{2}\right)
$$

where the phase is in the unit of degree. We define $45^{\circ}$ as the threshold of the phase difference. When the phase difference is less than $45^{\circ}, I P S$ is greater than 0.62 . We then calculate the ratio of the time with the IPS greater than 0.62 , termed phase synchrony index, PSI (Supplementary Fig. 5),

$$
P S I=\frac{t_{I P S>0.62}}{T}
$$

To determine the level of the phase synchrony, we categorize the two-dimensional scatter plot of PSI and PLV into four sections with both thresholds as 0.5: Zone 1 (low PSI and low PLV) indicates poor synchronization, Zone 2 (low PSI 
and high $P L V$ ) indicates medium synchronization, Zone 3 (high PSI and low $P L V$ ) indicates medium synchronization, and Zone 4 (high PSI and high PLV) indicates perfect synchronization (Supplementary Fig. 6).

Neural latent dynamic analysis. To characterize the latent dynamics associated with the recorded or reconstructed neural activity in each trial, we analyzed the filtered signals, which were obtained by applying a bandpass filter at $12.5 \mathrm{~Hz}$ and 30 $\mathrm{Hz}$, in the window starting at movement onset and ending $600 \mathrm{~ms}$ after movement onset. Such a window was selected was due to the interest in movement execution during the trial. For each trial, we obtained the data matrix $D$ of dimension $n$ by $T$, where $n$ is the number of recorded channels, $T$ is the number of time points in the given trial. Then we computed the low-dimensional manifold by applying principal component analysis (PCA) to $D$. The resulting PCs are the linear combination of measurements of all the channels. We would then rank these PCs based on the amount of neural variance explained by each PC explains. We keep only three leading PCs to represent the low-dimensional manifold, where these three leading PCs, or called neural modes, explain most of the variance in the data matrix (Supplementary Fig. 7).

Assuming the latent dynamics captured by the neural recordings and the reconstructed latent dynamics inferred from NBGNet's predictions are the projections on the different manifolds from the true latent dynamics ${ }^{45}$, we expect to identify the embedding space that true latent dynamics is located by using canonical correlation analysis (CCA). In CCA, given a pair of two latent trajectories, $\mathbf{P}_{A}$ and $\mathbf{P}_{B}$, linear transformations for each trajectory are identified to make the linearly transformed latent trajectories, $\widetilde{\mathbf{P}}_{A}$ and $\widetilde{\mathbf{P}}_{B}$, maximally correlated. First, $\mathrm{QR}$ decomposition is applied to both latent trajectories,

$$
\begin{aligned}
& \mathbf{P}_{A}^{T}=\mathbf{Q}_{A} \mathbf{R}_{A} \\
& \mathbf{P}_{B}^{T}=\mathbf{Q}_{B} \mathbf{R}_{B}
\end{aligned}
$$

Then the singular value decomposition is performed on the inner product of $\mathbf{Q}_{A}$ and $\mathbf{Q}_{B}$.

$$
\mathbf{Q}_{A}^{T} \mathbf{Q}_{B}=\mathbf{U S V}^{T}
$$

The transformation matrix, $\mathbf{T}_{A}$ and $\mathbf{T}_{B}$, is obtained by,

$$
\begin{aligned}
\mathbf{T}_{A} & =\mathbf{R}_{A}^{-1} \mathbf{U} \\
\mathbf{T}_{B} & =\mathbf{R}_{B}^{-1} \mathbf{V}
\end{aligned}
$$

Accordingly, the transformed latent trajectories are given by

$$
\begin{aligned}
\widetilde{\mathbf{P}}_{A}^{T} & =\mathbf{P}_{A}^{T} \mathbf{T}_{A} \\
\widetilde{\mathbf{P}}_{B}^{T} & =\mathbf{P}_{B}^{T} \mathbf{T}_{B}
\end{aligned}
$$

The correlation between the transformed latent trajectories, termed canonical correlation (CC), is obtained by the Pearson correlation coefficient. As CC is sorted from the largest to the smallest in CCA, we expect to observe a descending order from neural mode 1 to mode 3 .

Features selection for decoding the direction of the movement. We introduced several features per channel as candidates for the decoder and selected the leading number of features for further analysis. For each channel, we obtained total 34 features, including root mean square (RMS), mean frequency (MF), waveform length (WL), and the power at certain frequency ranged from $10-40 \mathrm{~Hz}$ (step size as $1 \mathrm{~Hz}$ ).

$$
\begin{gathered}
R M S=\sqrt{\frac{1}{T} \sum_{t=0}^{T-1} \mathbf{Y}(t)^{2}} \\
M F=\frac{\sum_{t=0}^{T-1} f_{t} p_{t}}{\sum_{t=0}^{T-1} p_{t}}
\end{gathered}
$$




$$
W L=\sum_{t=1}^{T-1}|\mathbf{Y}(t)-\mathbf{Y}(t-1)|
$$

where $\mathbf{Y}(t)$ represents the neural signals, $T$ is the number of time points in the given trial, $f_{t}$ and $p_{t}$ are the frequencies of the power spectrum and the corresponding amplitude.

To determine the features to be selected, we calculate the Fisher score for each candidate and select to the leading features. The Fisher score, $F\left(x^{i}\right)$, for the $i$-th feature, $x^{i}$, is computed by

$$
F\left(x^{i}\right)=\frac{\sum_{j=1}^{c} n_{j}\left(\mu_{j}^{i}-\mu^{i}\right)^{2}}{\sum_{j=1}^{c} n_{j}\left(\sigma_{j}^{i}\right)^{2}}
$$

where $\mu_{j}^{i}$ and $\sigma_{j}^{i}$ are the mean and standard deviation of the $j$-th class corresponding to the $i$-th feature, $\mu^{i}$ denotes the mean of the whole data set corresponding to the $i$-th feature, $n_{j}$ represents the size of the $j$-th class, and $c$ is the total number of classes. After computing the Fisher score for each feature, we selected the top-five ranked features to predict the subject's behavior. The features selected for analysis are summarized in Supplementary Table 3.

Predicting target direction from LFP and screw ECoG. To test whether the reconstructed activity from NBGNet maintain movement-related information, we built linear decoders to predict the direction of the movement based on the neural activity. Our hypothesis was that our NBGNet inference should provide as accurate predictions as the neural recordings. To test this hypothesis, we compared the predictive accuracy of seven types of decoders: (1) a decoder trained and tested based on screw ECoG; (2) a decoder trained and tested based on reconstructed screw ECoG inferred by forward-NBGNet; (3) a decoder trained and tested based on LFP; (4) a decoder trained and tested based on reconstructed LFP inferred by inverse-NBGNet; (5) a decoder trained and tested based on screw ECoG and LFP; (6) a decoder trained and tested based on reconstructed screw ECOG and LFP; and (7) a decoder trained and tested based on reconstructed LFP and screw ECoG. All decoders were linear support vector machine that used the selected features (Supplementary Table 3) as inputs to predict the direction of cursor's movement. They were trained and tested on the same day, using five-fold cross-validation procedure to protect against overfitting. Chance-level performance was obtained by shuffling the dataset. As expected, all the predictive accuracy was higher than the chance-level, which was around $12.5 \%$.

Comparison methods. To evaluate performance of NBGNet as compared to other existing algorithms, we implemented two approaches from different perspectives: electrophysiology-based sphere head model and data-driven recurrent neural network.

Sphere head model. Sphere head model is widely used to either compute the contribution from the current dipoles to the electrical potentials recorded at scalp electroencephalography (EEG) or estimate the current dipole sources based on the scalp potentials. Assume the head can be modeled as a four-layered sphere where each layer represents each tissue: brain, cerebrospinal fluid, skull, and scalp, respectively. Using the quasi-static approximation of Maxwell's equations and the volume-conductor theory, the electrical potential, $\boldsymbol{\Phi}(\mathbf{r}, t)$, can be calculated by the following Poisson equation ${ }^{46}$ :

$$
\nabla \cdot \sigma(\mathbf{r}) \nabla \boldsymbol{\Phi}(\mathbf{r}, t)=-C(\mathbf{r}, t)
$$

where $\sigma(\mathbf{r})$ represents the position-dependent conductivity of the medium, and $C(\mathbf{r}, t)$ is the density of the current sources. Assuming the conductivity to be isotropic, the solution provided by sphere head model to equation (36) is

$$
\begin{gathered}
\boldsymbol{\Phi}^{s+1}\left(\mathbf{r}_{s}, t\right)=\boldsymbol{\Phi}^{s}\left(\mathbf{r}_{s}, t\right) \\
\sigma_{s+1} \frac{\partial \boldsymbol{\Phi}^{s+1}\left(\mathbf{r}_{s}, t\right)}{\partial r}=\sigma_{s} \frac{\partial \boldsymbol{\Phi}^{s}\left(\mathbf{r}_{s}, t\right)}{\partial r}
\end{gathered}
$$




$$
\frac{\partial \Phi^{4}\left(\mathbf{r}_{4}, t\right)}{\partial r}=0
$$

where each layer is labeled by $s=1$ to 4 . Here we assume the dipole is radial with the magnitude $p(t)$ at location $r_{z}$, the analytical solution to equation ( $37-39)$ is given by,

$$
\begin{array}{cl}
\boldsymbol{\Phi}^{1}(r, \theta, t)=\frac{p(t)}{4 \pi \sigma_{1} r_{z}} \sum_{n=1}^{\infty}\left[A_{n}^{1}\left(\frac{r}{r_{1}}\right)^{n}+\left(\frac{r_{z}}{r}\right)^{n+1}\right] n P_{n}(\cos (\theta)) & r_{z}<r \leq r_{1} \\
\boldsymbol{\Phi}^{S}(r, \theta, t)=\frac{p(t)}{4 \pi \sigma_{1} r_{z}} \sum_{n=1}^{\infty}\left[A_{n}^{S}\left(\frac{r}{r_{s}}\right)^{n}+B_{n}^{S}\left(\frac{r_{s}}{r}\right)^{n+1}\right] n P_{n}(\cos (\theta)) & r_{s-1} \leq r \leq r_{s}
\end{array}
$$

where $\boldsymbol{\Phi}^{s}(r, \theta, t)$ is the extracellular potential measured at radius $r$ and the angle $\theta$ between measurement and dipole location vectors in the shell $s, r_{s}$ represents the radius of sphere $s, A_{n}^{s}$ and $B_{n}^{s}$ are the coefficients depending on the radius and conductivities of each medium, where are defined $\mathrm{in}^{47}$, and $P_{n}(\cos (\theta))$ represents the $n$-th Legendre Polynomial. As the solution is implemented into the case where we have $n_{d}$ current dipoles and $n_{r}$ recording units, a linear transformation matrix $F$ of dimension $n_{r}$ by $n_{d}$ can be obtained and utilized to convert the dipole moment vectors $\mathbf{X}$ into the electrical potential $\mathbf{Y}$, given by $\mathbf{Y}=F \mathbf{X}$. This is so-called forward mapping. When we performed inverse mapping to estimate $\mathbf{X}$ from $\mathbf{Y}$, we need to solve such an underdetermined system with pseudo-inverse by minimizing the following equation,

$$
\|\mathbf{X}\|^{2}+\lambda(\mathbf{Y}-F \mathbf{X})
$$

The solution to minimizing the equation (42) is given by,

$$
\mathbf{X}=F^{T}\left(F F^{T}\right)^{-1} \mathbf{Y}
$$

Here we segment the brain into 3600 segments $\left(n_{d}=3600\right)$, where each segment includes a potential current dipole source. Since our data for comparison does not include dipole sources, we adapt the algorithms into two step computation for both forward and inverse model. In forward model, we performed inverse mapping from LFP toward estimated dipole sources followed by a forward mapping from the estimated dipole sources toward screw ECoG recordings. Similarly, in inverse model, we performed inverse mapping from screw ECoG toward estimated dipole sources followed by a forward mapping from the estimated dipole sources toward LFP recordings. The parameters utilized are summarized in Supplementary Table 4.

Recurrent neural network (RNN). RNN is a commonly-used deep learning method to model a nonlinear dynamical system which includes three characteristics: nonlinearity, recurrent connection, and hidden dynamic states. In order to handle the long-term dependency embedded in the neural activity, Long Short-Term Memory (LSTM) ${ }^{48}$ is often implemented, where in each time point, model can determine the information to be forgotten, to be stored, or new information to be added. Two hidden layers with 64 and 16 nodes were connected to the output of LSTM unit, followed by the output layer. To make a fair comparison, the training details, including training iteration, the split ratio of training and validation data, and the choice of optimizer, were set to be the same as NBGNet.

\section{Reference}

43. Káli, S. \& Dayan, P. The Involvement of Recurrent Connections in Area CA3 in Establishing the Properties of Place Fields: a Model. J Neurosci 20, 7463-7477 (2000).

44. Sain, M. \& Massey, J. Invertibility of linear time-invariant dynamical systems. IEEE Transactions on Automatic Control 14, 141-149 (1969). 
bioRxiv preprint doi: https://doi.org/10.1101/20201130.404244. this version posted December 2 2020. The copyright holder for this preprint

(which was not certified by peer review) is the author/funder, who has granted bioRxiv a license to display the preprint in perpetuity. It is made available under aCC-BY-NC-ND 4.0 International license.

45. Gallego, J. A., Perich, M. G., Chowdhury, R. H., Solla, S. A. \& Miller, L. E. Long-term stability of cortical population dynamics underlying consistent behavior. Nature Neuroscience 23, 260-270 (2020).

46. Nunez, P. L. \& Srinivasan, R. Electric Fields of the Brain: The neurophysics of EEG. Electric Fields of the Brain (Oxford University Press, USA, 2006).

47. Næss, S. et al. Corrected Four-Sphere Head Model for EEG Signals. Front. Hum. Neurosci. 11, 490 (2017).

48. Hochreiter, S. \& Schmidhuber, J. Long Short-Term Memory. Neural Computation 9, 1735-1780 (1997). 